\title{
Multi-scale radio-infrared correlations in $M 31$ and $M$ 33: The role of magnetic fields and star formation
}

\author{
F. S. Tabatabaei ${ }^{1}$, E. M. Berkhuijsen ${ }^{2}$, P. Frick ${ }^{3}$, R. Beck ${ }^{2}$, and E. Schinnerer ${ }^{1}$ \\ 1 Max-Planck Institut für Astronomie, Königstuhl 17, 69117 Heidelberg, Germany \\ e-mail: taba@mpia.de \\ 2 Max-Planck Institut für Radioastronomie, Auf dem Hügel 69, 53121 Bonn, Germany \\ ${ }^{3}$ Institute of Continuous Media Mechanics, Korolyov str. 1, 614013 Perm, Russia
}

Received 30 January 2012 / Accepted 4 August 2013

\begin{abstract}
Interstellar magnetic fields and the propagation of cosmic ray electrons have an important impact on the radio-infrared (IR) correlation in galaxies. This becomes evident when studying different spatial scales within galaxies. We investigate the correlation between the IR and free-free/synchrotron radio continuum emission at $20 \mathrm{~cm}$ from the two local group galaxies M 31 and M 33 on spatial scales between 0.4 and $10 \mathrm{kpc}$. The multi-scale radio-IR correlations have been carried out using a wavelet analysis. The free-free and IR emission are correlated on all scales, but on some scales the synchrotron emission is only marginally correlated with the IR emission. The synchrotron-IR correlation is stronger in M 33 than in M 31 on small scales $(<1 \mathrm{kpc})$, but it is weaker than in M 31 on larger scales. Taking the smallest scale on which the synchrotron-IR correlation exists as the propagation length of cosmic ray electrons, we show that the difference on small scales can be explained by the smaller propagation length in M 33 than in M 31 . On large scales, the difference is due to the thick disk/halo in M 33, which is absent in M 31. A comparison of our data with data on NGC 6946, the LMC and M 51 suggests that the propagation length is determined by the ratio of ordered-to-turbulent magnetic field strength, which is consistent with diffusion of CR electrons in the ISM. As the diffusion length of CR electrons influences the radio-IR correlation, this dependence is a direct observational evidence of the importance of magnetic fields for the radio-IR correlation within galaxies. The star-formation rate per surface area only indirectly influences the diffusion length as it increases the strength of the turbulent magnetic field.
\end{abstract}

Key words. galaxies: ISM - galaxies: magnetic fields - galaxies: star formation - radio continuum: galaxies - infrared: galaxies galaxies: individual: M 33

\section{Introduction}

The correlation between radio and infrared (IR) continuum emission from galaxies is one of the tightest known in astronomy (e.g., Helou et al. 1985; de Jong et al. 1985; Gavazzi et al. 1986). The global radio-IR correlation is nearly linear and invariant for over four orders of magnitude in luminosity (e.g. Yun et al. 2001) and holds for all kinds of star forming galaxies (see Condon 1992, and references therein). The linearity of the correlation is conventionally attributed to a common dependence of IR and radio emission on massive star formation. Massive stars heat the dust and ionize the gas leading to thermal dust emission and thermal (free-free) radio emission. The same stars are progenitors of supernova remnants (SNRs) that produce cosmic ray electrons (CREs) radiating synchrotron emission. However, an important omission in this simple picture is the role of magnetic fields that determine the intensity of the synchrotron emission with about the square of the field strength. Lacki et al. (2010) modeled all relevant processes and found that the linearity of the global radio-IR correlation is due to a conspiracy of several factors (see also Bell 2003). For instance, in galaxies of low surface brightness and low star formation rate (SFR), the weak IR emission is balanced by weak radio emission caused by escape of CREs.
The radio-IR correlation also holds within galaxies, where local variations were found by several authors (e.g. Beck \& Golla 1988; Hoernes et al. 1998; Gordon et al. 2004; Hughes et al. 2006; Dumas et al. 2011; Tabatabaei et al. 2013). Detailed multi-scale analysis showed that the smallest scale on which the radio-IR correlation occurs is not the same in the LMC (Hughes et al. 2006) and M 51 (Dumas et al. 2011), the reason being unclear. Variations may be caused by differences in massive SF or dust heating sources, in magnetic field structure or in the propagation of cosmic ray electrons (CREs).

One fact making the IR-SF linkage less obvious is that the IR emission consists of at least two emission components, cold dust and warm dust. The cold dust emission may not be directly linked to the young stellar population as it is heated by the interstellar radiation field (ISRF, Xu 1990). Furthermore, it is necessary to distinguish between the two main radio continuum (RC) components, free-free and synchrotron emission. The free-free emission from electrons in HII regions around young, massive stars is expected to be closely connected to the warm dust emission that is heated by the same stars (e.g. Condon 1992). Although the CREs responsible for synchrotron emission also originate from SF regions (supernova remnants, the final stages in the evolution of massive stars), the synchrotron-IR correlation may not be as tight as the free-free-IR correlation locally, as a result of the propagation of CREs from their places of 
Table 1. Images of M 33 and M 31 used in this study.

\begin{tabular}{lcccc}
\hline \hline & \multicolumn{2}{c}{ M31 } & \multicolumn{2}{c}{ M33 } \\
Wavelength & Resolution & Telescope & Resolution & Telescope \\
\hline $20 \mathrm{~cm}$ & $45^{\prime \prime}$ & VLA+Effelsberg & $51^{\prime \prime}$ & VLA+Effelsberg \\
$160 \mu \mathrm{m}$ & $40^{\prime \prime}$ & Spitzer-MIPS & $40^{\prime \prime}$ & Spitzer-MIPS $^{4}$ \\
$70 \mu \mathrm{m}$ & $18^{\prime \prime}$ & Spitzer-MIPS & $18^{\prime \prime}$ & Spitzer-MIPS $^{3}$ \\
$24 \mu \mathrm{m}$ & $6^{\prime \prime}$ & Spitzer-MIPS $^{3}$ & $6^{\prime \prime}$ & Spitzer-MIPS $^{4}$ \\
$6563 \AA(\mathrm{H} \alpha)$ & $1.5^{\prime \prime}$ & KPNO $^{5}$ & $2^{\prime \prime}$ & KPNO $^{6}$ \\
\hline
\end{tabular}

Notes. ${ }^{(1)}$ Beck et al. (1998); ${ }^{(2)}$ Tabatabaei et al. (2007b); ${ }^{(3)}$ Gordon et al. (2006); ${ }^{(4)}$ Tabatabaei et al. (2007a); ${ }^{(5)}$ Devereux et al. (1994); ${ }^{(6)}$ Hoopes \& Walterbos (2000).

birth (see Longair 1994, and references therein). The CREs experience various energy losses interacting with matter and magnetic fields in the ISM, causing variation in the power law index of their energy distribution. A significant variation was found in M 33 by using a thermal radio tracer (TRT) method separating free-free and synchrotron emission that revealed flatter synchrotron spectra in regions of massive SF than in between the arms and in the outer disk (Tabatabaei et al. 2007b). This method yields more realistic free-free and synchrotron maps than the conventional separation based on a constant synchrotron spectral index.

As the variations in the radio-IR correlation are possibly due to a range of different conditions such as the SFR, heating sources of dust, magnetic fields, and CRE propagation, one needs to separate the free-free and synchrotron contributions to the RC emission in nearby galaxies that allow detailed, well resolved studies. Such a detailed study has been recently carried out in NGC 6946, which showed, for the first time, a direct link between the variation of the radio-IR correlation and the total magnetic field strength (Tabatabaei et al. 2013). In the present paper, we separate the free-free and synchrotron emission from the Andromeda galaxy using the same TRT method as for M 33 (Tabatabaei et al. 2007b). The influence of different star formation activities and ISM conditions on the radio-IR correlation is ideally traced by comparing early-type and late-type spirals like the nearest galaxies M $31(D=780 \mathrm{kpc}$, Stanek \& Garnavich $1998)$ and M 33 ( $D=840 \mathrm{kpc}$, Freedman et al. 1991). The free-free and synchrotron emission maps of M 33 and M 31 enable us to separately compare the free-free-IR correlation and synchrotron-IR correlation in these galaxies.

In this paper, we analyze the radio-IR correlation scaleby-scale rather than using classical pixel-by-pixel correlations. The usual pixel-by-pixel correlation between the distributions of FIR and radio emission contains all scales that exist in a galaxy image. For example, the high-intensity points represent high-emission peaks at small scales belonging to bright sources, whereas low-intensity points generally represent weak diffuse emission with a large-scale distribution.

A scale-by-scale approach is required for an unbiased recognition of similarities and differences between images tracing different emission mechanisms. Such a study enables us to uncover the scale dependence of the correlation of emitting structures. This method helps to identify the smallest scale where the radioFIR correlations break down, and provides important information on the propagation length of CREs.

To separate the contribution from different spatial scales in the global correlation of two maps Frick et al. (2001) introduced the "wavelet cross-correlation". The wavelet transform, similar to the Fourier transform, decomposes the images scale-by-scale, but, in contrast to the Fourier decomposition, wavelets keep the information about the distribution of structures of a given scale in the physical space and therefore allow one to analyse the correlation of two fields scale-by-scale.

We describe the relevant data sets in Sect. 2. The separation of the RC maps in free-free and synchrotron components in M 31 is explained in Sect.3. Wavelet decompositions and wavelet spectra of the radio and IR emission, and their cross correlations are presented in Sect. 4. We discuss and summarize our results in Sects. 5 and 6, respectively.

\section{Data}

Table 1 summarizes the data used in this work. The interferometeric observations of M 31 and M 33 at $20 \mathrm{~cm}$ were made with the Very Large Array $\left(\mathrm{VLA}^{1}\right)$. Single-dish observations at the same wavelength were carried out with the 100-m Effelsberg telescope ${ }^{2}$. We used the combined VLA + Effelsberg $20 \mathrm{~cm}$ radio continuum data of Beck et al. (1998) for M 31 and of Tabatabaei et al. (2007c) for M 33.

We used the Spitzer-MIPS data at $24 \mu \mathrm{m}, 70 \mu \mathrm{m}$, and $160 \mu \mathrm{m}$ for M 31 (Gordon et al. 2006) and M 33 (Tabatabaei et al. 2007a). The basic data reduction and mosaicing was performed with the MIPS instrument Data Analysis Tool Version 2.90 (Gordon et al. 2005). For both galaxies, the sky and background sources in the MIPS maps were subtracted as detailed in Gordon et al. (2006) and Tabatabaei et al. (2007a).

The $\mathrm{H} \alpha$ observations of M 31 were carried out on the Case Western Burrell-Schmidt telescope at the Kitt Peak National Observatory, covering a field of view of $2^{\circ} \times 2^{\circ}$ for M 31 (Devereux et al. 1994) and of $68^{\prime} \times 68^{\prime}$ for M 33 (Hoopes \& Walterbos 2000). Here, we use the de-reddened $\mathrm{H} \alpha$ maps of M 31 and M 33 presented in Tabatabaei \& Berkhuijsen (2010) and Tabatabaei et al. (2007b), respectively.

For M 31, we subtracted background radio sources with flux densities $S>35 \mathrm{mJy}$ from the combined $20 \mathrm{~cm}$ radio map ${ }^{3}$. Furthermore, the bright nucleus of M 31 was subtracted from all radio and IR maps before the wavelet analysis, as it causes a strong bias in our wavelet results. For M 33, all known background radio sources given by Viallefond et al. (1986) were subtracted. The MIPS maps were convolved to the resolution of the VLA $20 \mathrm{~cm}$ radio data (45" for M 31 and 51" for M 33) by using the custom kernels presented by Gordon et al. (2007). After

1 The VLA (Jansky VLA) is a facility of the National Radio Astronomy Observatory. The NRAO is operated by Associated Universities, Inc., under contract with the National Science Foundation. 2 The 100-m telescope at Effelsberg is operated by the Max-PlanckInstitut für Radioastronomie (MPIfR) on behalf on the Max-PlanckGesellschaft.

3 Any point source brighter than $35 \mathrm{mJy}$ in M 31 is too bright to be an SNR. 


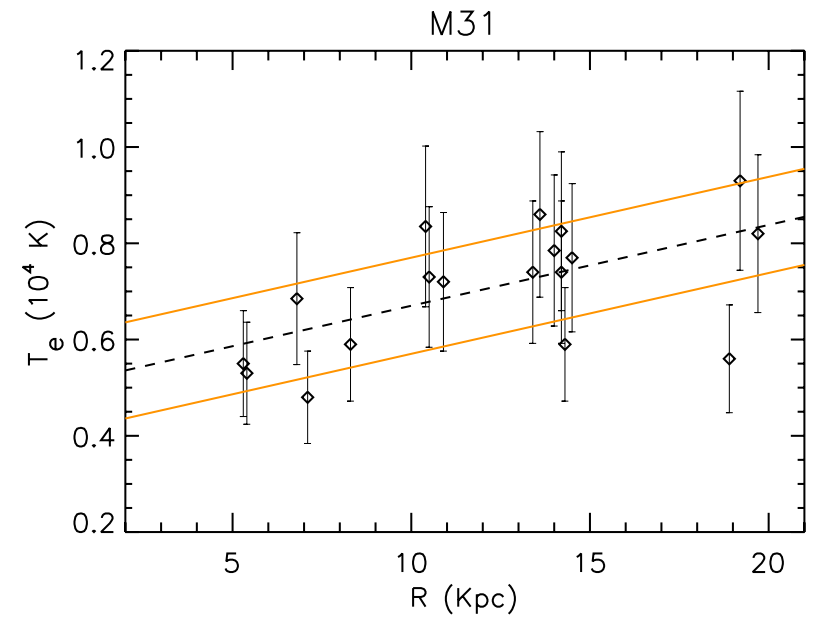

Fig. 1. Electron temperature estimates for M 31, based on the empirical method, the $([\mathrm{OII}]+[\mathrm{OIII}]) / \mathrm{H} \beta$ ratio, by Dennefeld \& Kunth (1981) and Blair et al. (1982) after correcting for M 31's inclination of $75^{\circ}$ and scaling to $D=780 \mathrm{kpc}$. Also shown are the fitted least-squares slope (dashed line) and the $5 \sigma$ confidence levels (red solid lines).

convolution, the maps were normalized in grid size, orientation and reference coordinates.

\section{Free-free and synchrotron emission}

We separate free-free and synchrotron emission from M31 and M 33 using the TRT method in which one of the hydrogen recombination lines is used as a template for the free-free emission (see e.g. Dickinson et al. 2003; Tabatabaei et al. 2007b). The brightest recombination line data available, the $\mathrm{H} \alpha$ line emission, is used for both galaxies. This pixel-by-pixel separation results in maps of the free-free and synchrotron emission for M 31 and M 33.

\section{1. $M 31$}

We use the de-reddened $\mathrm{H} \alpha$ emission presented in Tabatabaei \& Berkhuijsen (2010) and convert it to the emission measure via the expression of Valls-Gabaud (1998)

$I_{\mathrm{H} \alpha}=9.41 \times 10^{-8} T_{\mathrm{e} 4}^{-1.017} 10^{-\left(0.029 / T_{\mathrm{e} 4}\right)} \mathrm{EM}$,

where the electron temperature, $T_{\mathrm{e} 4}$, is in units of $10^{4} \mathrm{~K}$, EM in $\mathrm{cm}^{-6} \mathrm{pc}$, and it is assumed that the optical depths of HI resonance lines are large (usually denoted as case B). Optical spectroscopic observations of the HII regions in M 31 indicate that the electron temperature $\left(T_{\mathrm{e}}\right)$ increases with galactocentric radius as a result of the metallicity gradient (Dennefeld \& Kunth 1981; Blair et al. 1982). Figure 1 shows the $T_{\mathrm{e}}$ measurements of these authors obtained from the $([\mathrm{OII}]+[\mathrm{OIII}]) / \mathrm{H} \beta$ ratios. A least-squares fit to their results gives

$T_{\mathrm{e} 4}=(0.017 \pm 0.005) R+(0.5 \pm 0.1)$,

with $R$ the galactocentric radius in kpc, scaled to $D=780 \mathrm{kpc}$ and corrected for the inclination of $75^{\circ}$ before fitting the above line. The Galactic measurements of Madsen et al. (2006) give an average electron temperature $T_{\mathrm{e}}$ of about $1000 \mathrm{~K}$ higher in diffuse ionized gas (DIG) than in HII regions. Assuming that the same difference occurs in $\mathrm{M} \mathrm{31}$, and taking an HII region (DIG) contribution of $60 \%(40 \%)$ to the total $\mathrm{H} \alpha$ emission (e.g. Greenawalt et al. 1997), we derived the EM from Eqs. (1)
Table 2. Thermal fractions of the radio continuum emission, $F_{\text {th }}$, in M 31 at $20 \mathrm{~cm}$.

\begin{tabular}{lllll}
\hline \hline $\begin{array}{l}R \\
(\mathrm{kpc})\end{array}$ & $\begin{array}{l}R \\
(')\end{array}$ & $\begin{array}{l}S_{\text {obs }} \\
(\mathrm{mJy})\end{array}$ & $\begin{array}{l}S_{\text {th }} \\
(\mathrm{mJy})\end{array}$ & $\begin{array}{l}F_{\text {th }} \\
\%\end{array}$ \\
\hline $0-16$ & $0-70$ & $4000 \pm 400$ & $630 \pm 60$ & $16 \pm 2$ \\
$6.8-12.5$ & $30-55$ & $2600 \pm 300$ & $380 \pm 40$ & $15 \pm 2$ \\
$0-6.8$ & $0-30$ & $660 \pm 70$ & $200 \pm 20$ & $30 \pm 4$ \\
$0-1$ & $0-4.4$ & $120 \pm 10$ & $31 \pm 3$ & $26 \pm 3$ \\
\hline
\end{tabular}

Notes. Integrated flux density of the total $\left(S_{\text {obs }}\right)$ and thermal $\left(S_{\text {th }}\right)$ radio continuum emission at $20 \mathrm{~cm}$ for various radial intervals in the galactic plane (inclined by $75^{\circ}$ ). A distance of $780 \mathrm{kpc}$ was used for these calculations. Errors are systematic errors due to uncertainty in the zerolevel.

and (2). The EM is further related to the continuum optical thickness, $\tau_{\mathrm{c}}$, of the ionized gas by

$\tau_{\mathrm{c}}=8.235 \times 10^{-2} a T_{\mathrm{e}}^{-1.35} v_{\mathrm{GHz}}^{-2.1}(1+0.08) \mathrm{EM}$,

with $a \simeq 1$ (Dickinson et al. 2003). The factor $(1+0.08)$ takes the contribution from singly ionized He into account. The brightness temperature of the radio continuum emission, $T_{\mathrm{b}}$, then follows from

$T_{\mathrm{b}}=T_{\mathrm{e}}\left(1-\mathrm{e}^{-\tau_{\mathrm{c}}}\right)$

The resulting distribution of the intensity of the predicted freefree emission in Jy/beam at $20 \mathrm{~cm}$ is shown in Fig. 2 (top panel). Strong thermal emission emerges from the center of the galaxy with a maximum intensity of $\simeq 4 \mathrm{mJy} /$ beam and from complexes of star-forming regions that are most abundant in the north-east of the star-forming ring (the so-called " $10 \mathrm{kpc}$ ring", $30^{\prime}<R<$ $\left.55^{\prime}\right)$. By integrating the maps of the observed radio continuum and the free-free emission in rings around the galaxy center out to a radius of $16 \mathrm{kpc}$, we obtain the thermal flux density and thermal fraction (Table 2). The thermal fraction is $15 \% \pm 2 \%$ in the " $10 \mathrm{kpc}$ ring" and $30 \% \pm 4 \%$ for $R<30$ ' (6.8 kpc). The thermal flux density of $S_{\text {th }}=630 \pm 60 \mathrm{mJy}$ is lower than the value of $0.8 \pm 0.1 \mathrm{Jy}$ obtained by Hoernes et al. (1998), who used a constant nonthermal spectral index to separate the thermal and nonthermal components. A similar overestimate of the thermal emission was found in M 33 assuming a constant nonthermal spectral index (the so called standard thermal/nonthermal separation method, Tabatabaei et al. 2007b). This is due to the synchrotron emission being underestimated when the nonthermal spectral index is flatter than the one assumed in the standard method.

Possible origins of the strong $\mathrm{H} \alpha$ emission and high thermal fraction at $R<30^{\prime}$ have been discussed by Devereux et al. (1994) and Tabatabaei \& Berkhuijsen (2010). As recent SF is scarce in this region, the gas must have been ionized by shocks, evolved stars (Groves et al. 2012) and/or during the last encounter with a companion galaxy (Block et al. 2006).

Subtracting the free-free emission from the observed radio emission at $20 \mathrm{~cm}$, we derive the distribution of the synchrotron emission (Fig. 2, bottom). This map exhibits a wide ring of diffuse emission coinciding with the " $10 \mathrm{kpc}$ ring". Interestingly, the synchrotron emission is stronger close to star-forming regions. Some of these regions are accompanied by patches of relatively high-intensity ( $\gtrsim 2 \mathrm{mJy} /$ beam) synchrotron radiation. The strongest synchrotron emission emerges from the center of the galaxy with a maximum of $9 \mathrm{mJy} / \mathrm{beam}$. The extended, diffuse structure around the spiral arms indicates that CREs propagated away from their places of origin. 

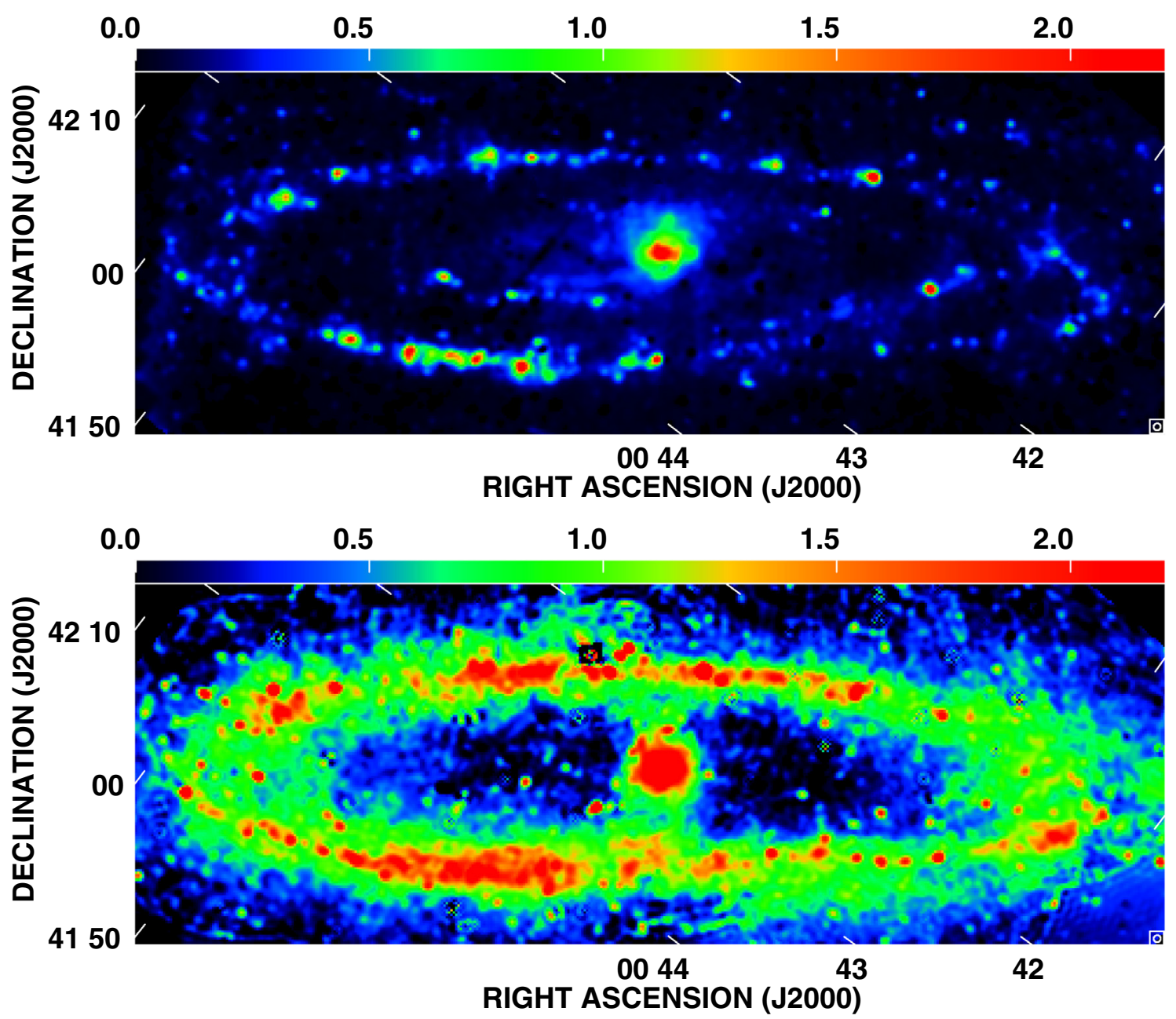

Fig. 2. Thermal free-free (top) and nonthermal synchrotron emission (bottom) from M 31 at $20 \mathrm{~cm}$, separated using the TRT method (Tabatabaei et al. 2007b). The color scale gives the flux density in mJy/beam. The angular resolution is 45" (shown in the lower right of the images) corresponding to $\simeq 180 \mathrm{pc}$ linear resolution along the major axis.

The high thermal fraction of about $30 \%$ at $R<30^{\prime}(6.8 \mathrm{kpc})$ is partly due to the weak synchrotron emission from regions outside the central region $(2.5<R<4.5 \mathrm{kpc})$ with a minimum of $240 \mu \mathrm{Jy} /$ beam (about 3 times the noise level). This is the result of a lack of cosmic ray electrons, since there are magnetic fields but there is very little recent star formation in this region (Moss et al. 1998; Berkhuijsen \& Humphreys 1989).

\section{2. $M 33$}

We presented the free-free and synchrotron maps of M 33 in Tabatabaei et al. (2007b) at 90" angular resolution. In this paper, for a proper comparison with $\mathrm{M} 31$, we re-derive the freefree and synchrotron maps at the resolution of the $20 \mathrm{~cm}$ VLA map, 51" (Tabatabaei et al. 2007a) (Fig. 3). The free-free map is dominated by HII regions, which are better resolved at 51" resolution compared to the results reported by Tabatabaei et al. (2007b). Moreover, smooth synchrotron spiral arms are detected with intensities of $\lesssim 2 \mathrm{mJy} /$ beam $(\simeq 10 \sigma)$, which were not visible at $90^{\prime \prime}$ resolution due to beam smearing. The strong synchrotron clumps close to the sites of active star forming regions shown in Tabatabaei et al. (2007b) exhibit several sub-structures at 51" resolution. The general distributions of the synchrotron emission and the thermal fraction, however, remain the same as before.
Most of the distinct point sources coincide with supernova remnants, as shown in Tabatabaei et al. (2007b).

\section{Wavelet analysis and scale-by-scale correlation}

The next step of our analysis is the scale-by-scale comparison of the radio and IR emission from M 31 and M 33. We first describe the basics of the wavelet decomposition, then apply this method to decompose the radio and IR emission from each galaxy.

\subsection{Algorithms}

To illustrate why a scale-by-scale analysis can be useful we present in Fig. 4 an example. Two artificial maps are shown in this figure, both include four large-scale spots formed by a set of small-scale spots. The large-scale spots are located at the same coordinates, but the small-scale spots in one map are shifted to in between the small-scale spots in the other map. The two maps look very similar, but their cross-correlation

$$
c=\frac{\int\left(f_{1}(x)-\overline{f_{1}}\right)\left(f_{2}(x)-\overline{f_{2}}\right) \mathrm{d} x}{\left(\int\left(f_{1}(x)-\overline{f_{1}}\right)^{2} \mathrm{~d} x \int\left(f_{2}(x)-\overline{f_{2}}\right)^{2} \mathrm{~d} x\right)^{1 / 2}}=0.03
$$

is weak (here $f_{1}$ and $f_{2}$ are the intensities of both maps, and $\overline{f_{1}}$ and $\overline{f_{2}}$ denote averages). 

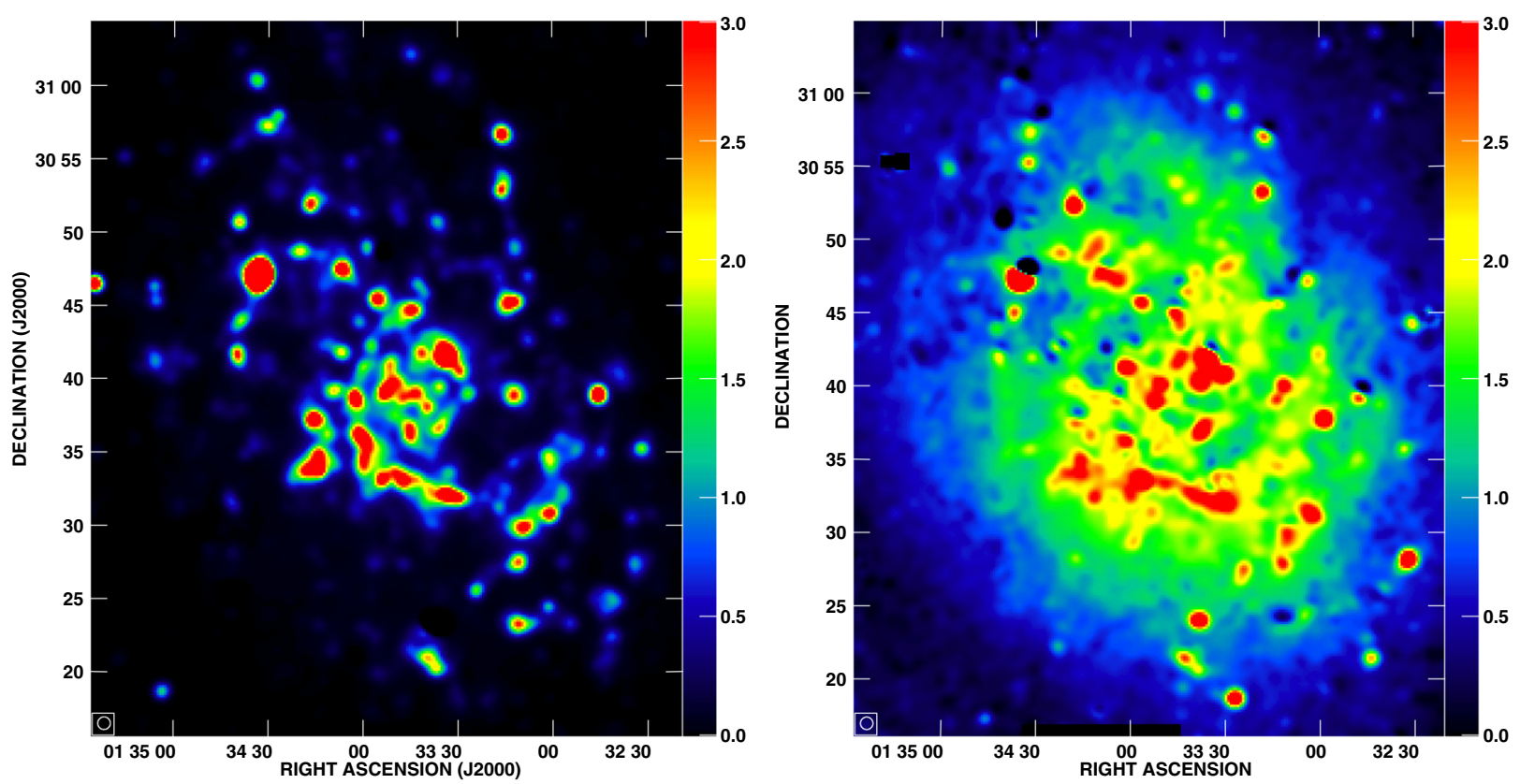

Fig. 3. Thermal (left) and synchrotron (right) radio continuum emission from M 33 at $20 \mathrm{~cm}$ at 51" resolution, separated using the TRT method (Tabatabaei et al. 2007b). The color scale gives the flux density in $\mathrm{mJy} / \mathrm{beam}$.
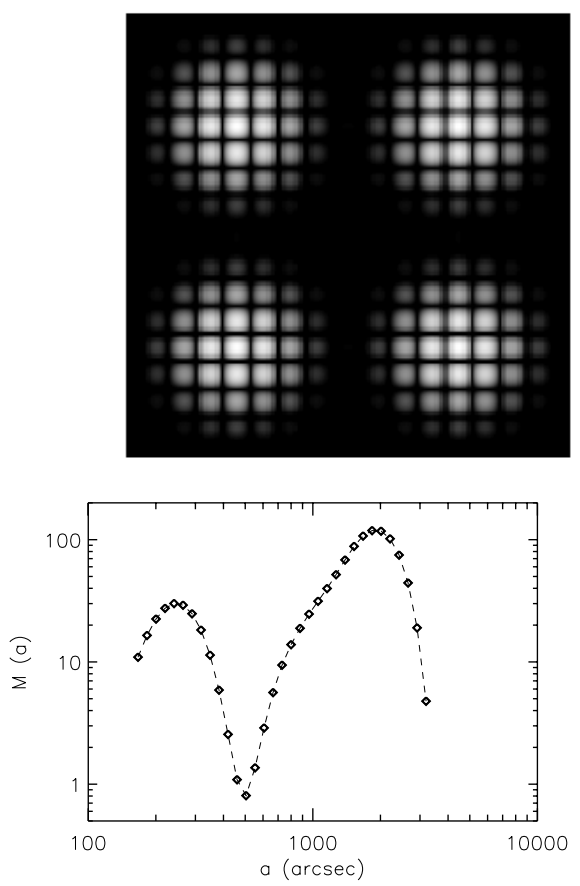
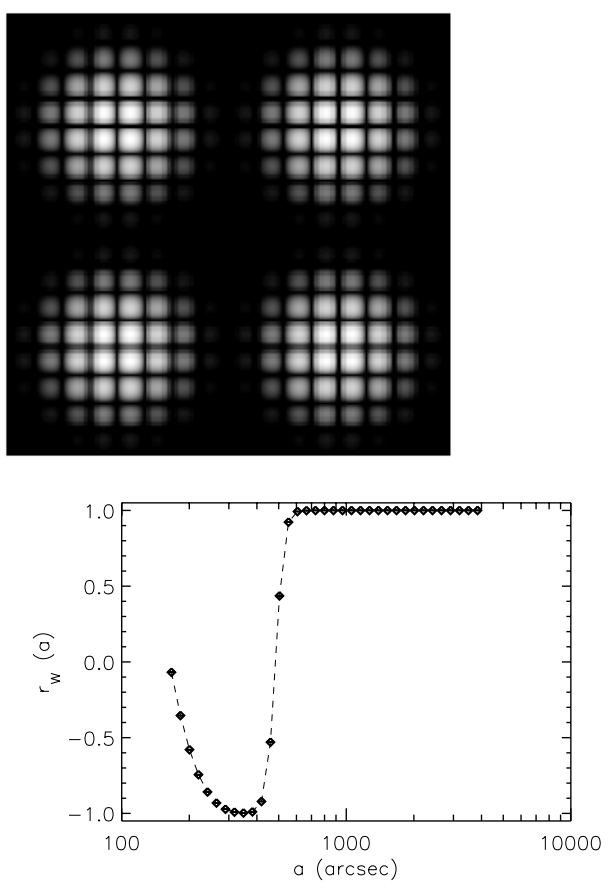

Fig. 4. Artificial example of a scale-by-scale wavelet cross-correlation analysis. Top: two different mock maps before the wavelet decomposition. The maps show four large-scale spots made by small-scale spots. The largescale spots in the two maps slightly differ in size ( 6 rows and columns in the right panel, 5 rows and columns in the left panel). As they are centered at the same location, the smallscale spots are shifted with respect to each other. Bottom left: wavelet spectra (they are identical for both maps), bottom right: wavelet cross-correlation function.
For this simple example it is obvious that the large scales are completely correlated and the small scales are anti-correlated. For less obvious cases one needs some formal algorithm to separate different levels of correlation on different scales. The algorithm should include two steps: first, some scale filtering, which isolates the structures in a given scale range, and second, calculation of correlation in filtered maps. We note that Fourier filtering is of limited use in this situation because each Fourier mode covers the whole physical space and leaves no possibility for statistics over the map. Instead, one needs a scale filtering which keeps the independency of structures in physical space, i.e. filtering, localized in both Fourier and physical space. This is the underlying idea of the wavelet transform which makes a spatial-scale decomposition using the convolution of the data with a family of self-similar basic functions that depend on the scale and the location of the structure. The wavelet coefficients for the $2 \mathrm{D}$ continuous wavelet transform are given by:

$W(a, x)=\frac{1}{a^{\kappa}} \int_{-\infty}^{+\infty} \int_{-\infty}^{+\infty} f\left(x^{\prime}\right) \psi^{*}\left(\frac{x^{\prime}-x}{a}\right) \mathrm{d} x^{\prime}$,

where $f(x)$ is the analyzed, two-dimensional function (the image), $\psi(x)$ is the analyzing wavelet, the symbol * denotes the complex conjugate, $x=(x, y)$ defines the position of the wavelet, $a$ defines its scale, and $\kappa$ is the normalization parameter. In Fourier space, the wavelet coefficients (Eq. (5)) can be expressed as

$W(a, x)=\frac{a^{2-\kappa}}{4 \pi^{2}} \int_{-\infty}^{+\infty} \int_{-\infty}^{+\infty} \hat{f}(k) \psi^{*}(a k) \mathrm{e}^{\mathrm{i} k x} \mathrm{~d} k$ 
where $\hat{f}(k)$ is Fourier transform of the function $f(x)$ and $k=$ $\left(k_{x}, k_{y}\right)$ is the wavevector. Equations (5) and (6) allow one to plot a $2 \mathrm{D}$ map for a given scale $a$ (to be more precise, for a range of scales $a \pm \Delta a / 2$, where $\Delta a$ is the scale resolution of the analyzing wavelet).

The choice of the analyzing wavelet $\psi$ is very important and strongly depends on the goal of the performed analysis. For the scale-by-scale cross-correlation analysis one needs a good scale resolution $\Delta a$ (to separate the contribution from different spatial scales) and a good spatial resolution $\Delta x$ (to increase the number of independent areas). These two requirements are mutually exclusive $(\Delta x \Delta a=2 \pi)$. A reasonable compromise for the crosscorrelation analysis of 2D maps has been suggested by Frick et al. (2001), who used the wavelet function called "Pet Hat", first introduced in Aurell et al. (1994) for turbulent flow modeling. The Pet-Hat function is defined in Fourier space by the formula

$$
\psi(k)=\left\{\begin{array}{lr}
\cos ^{2}\left(\frac{\pi}{2} \log _{2}\left(\frac{k}{2 \pi}\right)\right) & \pi \leq k \leq 4 \pi \\
0 & k<\pi \text { or } k>4 \pi,
\end{array}\right.
$$

where $k$ is the wavevector and $k=|k|$. This function has been used by several authors to study the scale distribution of various emission mechanisms in galaxies (e.g. Frick et al. 2001; Hughes et al. 2006; Laine et al. 2010; Dumas et al. 2011). 2D continuous Pet-Hat transformations of the Spitzer-MIPS maps at 24, 70, and $160 \mu \mathrm{m}$ were presented for M 33 by Tabatabaei et al. (2007a) and for M 31 by Tabatabaei \& Berkhuijsen (2010).

Similar to the standard Fourier energy spectrum given by $E(k)=\int_{|k|}|\hat{f}(k)|^{2} \mathrm{~d} k$, the wavelet energy spectrum, i.e. the scale distribution of the emission is defined as the energy in all wavelet coefficients of scale $a$,

$M(a)=\int_{-\infty}^{+\infty} \int_{-\infty}^{+\infty}|W(a, x)|^{2} \mathrm{~d} x$,

which is expressed in Fourier space as

$M(a)=\frac{a^{4-2 \kappa}}{16 \pi^{4}} \int_{-\infty}^{+\infty} \int_{-\infty}^{+\infty}|\hat{f}(k)|^{2}|\hat{\psi}(a k)|^{2} \mathrm{~d} k$.

We use $\kappa=2$, which provides the same power law for the wavelet spectrum as for the conventional second-order structure function (Frick et al. 2001). This selection also ensures a consistent comparison with previous studies (e.g. Hughes et al. 2006; Tabatabaei et al. 2007a; Dumas et al. 2011).

By decomposing the images into maps containing the structures of a given scale we can analyze the cross-correlation of the analyzed maps scale-by-scale (Nesme-Ribes et al. 1995; Frick et al. 2001). The wavelet cross-correlation coefficient at scale $a$ is defined for $2 \mathrm{D}$ maps as

$r_{w}(a)=\frac{\iint W_{1}(a, x) W_{2}^{*}(a, x) \mathrm{d} x}{\left[M_{1}(a) M_{2}(a)\right]^{1 / 2}}$,

where the subscripts refer to two images of the same size and same resolution. The value of $r_{w}$ varies between -1 (perfect anticorrelation) and +1 (perfect correlation). We will consider $\left|r_{w}\right|=0.5$ as a marginal value for the acceptance of a correlation between the structures of given scale ${ }^{4}$. Plotting $r_{w}$ against scale shows how well structures at different scales are correlated in intensity and location.

4 Generally, $\left|r_{w}\right|=0.5$ indicates the same number of correlated and uncorrelated structures in two decomposed maps.
The error of an estimated correlation depends on the number of independent points used, $n$, and on the degree of correlation (Edwards 1979)

$\Delta r_{w}(a)=\frac{\sqrt{1-r_{w}^{2}}}{\sqrt{n-2}}$,

where the number of independent points is estimated as $n \simeq$ $L_{x} L_{y} / a^{2}$ and $\left(L_{x}, L_{y}\right)$ are the map sizes in $(x, y)$. Thus, towards larger scales, $n$ decreases and the errors increase. For example, considering the size of our radio and IR maps for M 33, less than 5 independent points exist for scales $a>20^{\prime}$. After calculating the correlations, the t-student value $t=r_{w} \sqrt{(n-2) /\left(1-r_{w}^{2}\right)}$ (e.g. Hoernes et al. 1998) is smaller than 2 for $a>20^{\prime}$, which means that the correlation is not statistically significant. Therefore, we only consider wavelet correlations for scales smaller than $20^{\prime}$.

To illustrate what kind of information can be deduced from wavelet cross-correlation analysis we return to our test example and show in Fig. 4 the wavelet spectrum of the test image and the wavelet cross-correlation function. The spectrum shows two maxima corresponding to the scale of small spots and to the scale of a cloud of spots. In general, the analysis of the correlations $r_{w}(a)$ only makes sense for energy-containing scales, i.e. in the vicinity of the two peaks in the example discussed.

Actually, $r_{w} \approx-1$ near the first peak (the scale of small spots, (their locations differ in both maps) and $r_{w} \approx 1$ near the second peak (the location of the cloud of spots in both maps is the same).

Note that by using a continuous wavelet transform we formally can consider a continuous range of scales (or a set of scales with arbitrary small steps). However, the scale resolution of the wavelet is finite, which means that the adjacent scales are not independent and it is not correct to take a very dense sampling in scales. Actually, the wavelet (Eq. (7)) used below covers an octave in Fourier space, which means that a reasonable sampling corresponds to a scale doubling at each step. The samples used below are close to this criterion.

\subsection{Wavelet spectra and cross-correlations for $M 31$ and $M 33$}

We decomposed the $20 \mathrm{~cm}$ radio and the MIPS IR maps of the two galaxies into 8 angular scales $(a)$. Before decomposition, the central region $(R<1 \mathrm{kpc})$ in the M 31 maps was subtracted to avoid a strong influence of the nucleus on the results.

The decomposed maps of the synchrotron, free-free, and $160 \mu \mathrm{m}$ emissions are shown in Figs. 5 and 6 for M 31 and Fig. 7 for $\mathrm{M} 33$, on the smallest scale of $1.7^{\prime} \simeq 0.4 \mathrm{kpc}$ (about twice the beamwidth of the observed maps) and on a selected large scale of $a=12.5^{\prime}(\simeq 3 \mathrm{kpc})$ for M 33 and $a=11^{\prime}(\simeq 2.5 \mathrm{kpc})$ for M 31. On the smallest scale, resolved sources (e.g. HII regions) appear as distinct point-like structures, but narrow features of diffuse emission are also visible. On $a \simeq 3 \mathrm{kpc}$, only bright largescale (extended) structures are exhibited.

In $\mathrm{M}$ 31, a wide distribution of small-scale structures (see Fig. 5) occurs in the synchrotron map at the position of the " $10 \mathrm{kpc}$ ring", while the free-free emission is dominated by "points" coinciding with SF regions (they are at the same positions as the SF regions in Fig. 2, top). At $160 \mu \mathrm{m}$, the small-scale structures, are aligned along the spiral arms and are bright in the SF regions (see also the composite image in Fig. 5). The fact that the small-scale synchrotron emission does not follow the narrow arms indicates that CREs propagated away from their origin 

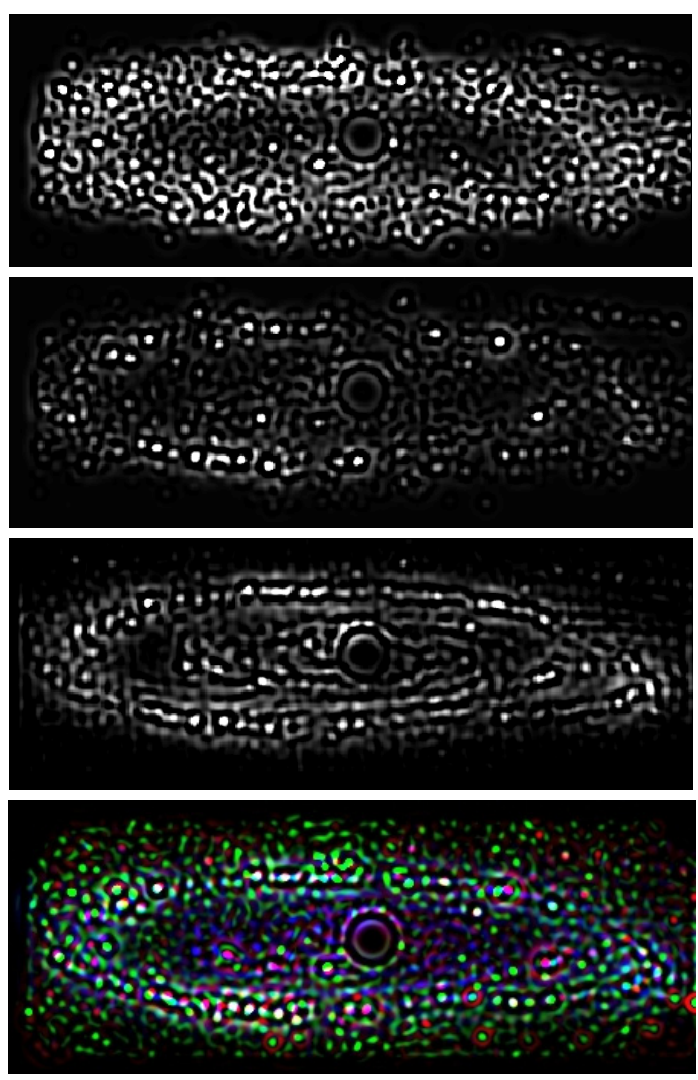

Fig. 5. M 31 maps of the synchrotron $(20 \mathrm{~cm}$, top $)$, free-free $(20 \mathrm{~cm}$, middle), and $160 \mu \mathrm{m}$ (bottom) emission on the smallest scale of the wavelet decomposition, $a=1.7^{\prime} \simeq 0.4 \mathrm{kpc}$ in arbitrary units. The emission from the central $2 \mathrm{kpc}$ was removed before decomposition. The map size is $110^{\prime} \times 38.5^{\prime}$. Also shown is the composite map (red: free-free, green: synchrotron, and blue: $160 \mu \mathrm{m}$ emission).

(see also Fig. 2, bottom). On the large scale of $\simeq 3 \mathrm{kpc}$ (Fig. 6), the wide " $10 \mathrm{kpc}$ ring" is now dominant in all emissions.

In M 33, the small-scale synchrotron emission is widely distributed across the entire disk, yet it is bright near the SF regions that are well visible in the free-free and the $160 \mu \mathrm{m}$ decomposed maps (Fig. 7, upper row). On the large scale of $\simeq 3 \mathrm{kpc}$, the two bright structures ${ }^{5}$ in the free-free and $160 \mu \mathrm{m}$ emission, seen near the center and on the position of the HII region NGC 604, are not visible in the synchrotron emission (Fig. 7, bottom row).

Hence, the morphological difference between the FIR $160 \mu \mathrm{m}$ and the synchrotron emission is more evident on the small scale of $0.4 \mathrm{kpc}$ than on the large scale of $3 \mathrm{kpc}$ in M 31, while it is the other way around in M 33. Generally, the FIR emission has a morphology more similar to that of the free-free than of the synchrotron emission in both galaxies.

To investigate which spatial scales dominate the emissions, we plot the wavelet spectra $M(a)$ versus spatial scale ${ }^{6}$ for the MIPS IR bands and the $20 \mathrm{~cm}$ radio emission in Fig. 8. In general, the presence of strong point-like sources causes a maximum on small scales. In case of a dominant component of extended diffuse emission, the spectrum increases towards larger scales.

Their adjacent dark regions are due to the negative part of the wavelet function applied.

6 Since $M(a)$ is a relative measure, standard deviations cannot be computed. The systematic effect of noise on small scales (Dumas et al. 2011), probed by adding Gaussian noise to the maps before decomposition, is negligible for M 31 and M 33 on scales shown in Fig. 8.
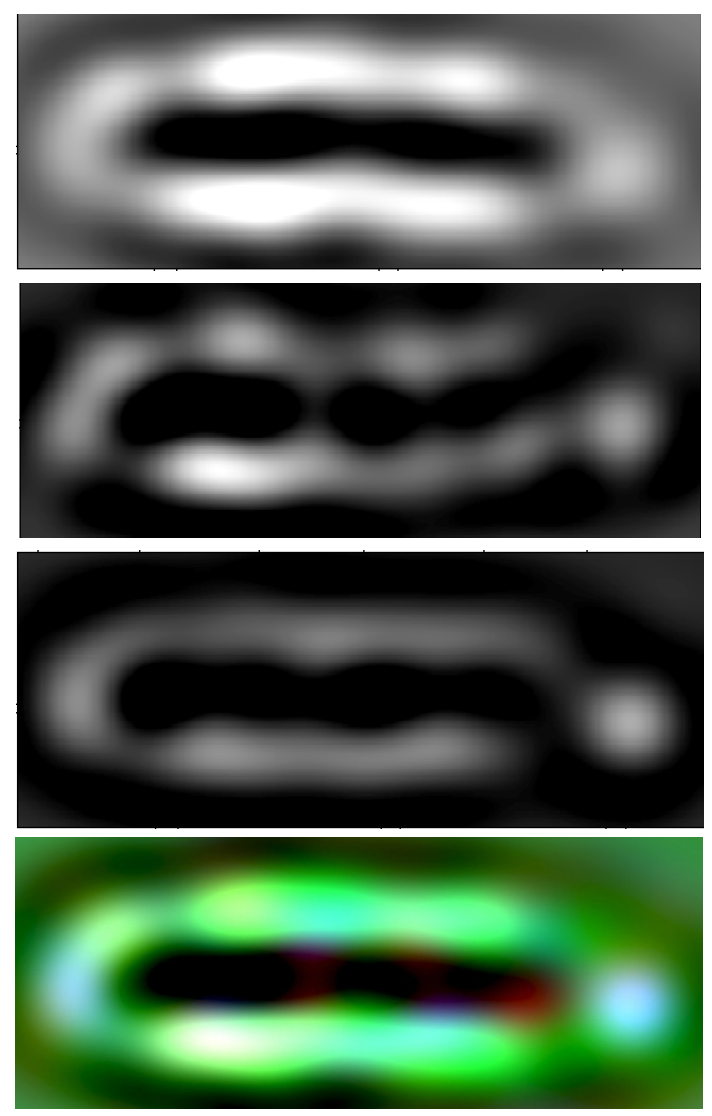

Fig. 6. Same as Fig. 5 for a large scale of $a=11^{\prime} \simeq 2.5 \mathrm{kpc}$.

In M 31 (Fig. 8, upper panels), the observed RC emission peaks at $a \simeq 4 \mathrm{kpc}$, which is the width of the bright synchrotron ring at $R \simeq 10 \mathrm{kpc}$. For the synchrotron emission small scales are less important than the larger scales, unlike for the freefree emission. The free-free emission is dominated by sources on $a \leq 2 \mathrm{kpc}$, residing mainly in M 31's " $10 \mathrm{kpc}$ ring". Among the IR bands, the $70 \mu \mathrm{m}$ spectrum is most similar to that of the free-free emission. The $160 \mu \mathrm{m}$ emission is entirely dominated by large scales indicating the importance of the ISRF in heating the cold dust.

M 33 shows a different behavior. The wavelet spectra of the $24 \mu \mathrm{m}$ and the free-free emission are most similar (Fig. 8, lower panels). Here even the cold dust emits significantly on small scales. Moreover, the radio emission, and even its synchrotron component, is relatively important on small scales. Comparing the (Fourier) spectral energy densities in the band $0.4<a<$ $2 \mathrm{kpc}$ in the two galaxies, we find that the synchrotron emission on these scales is a factor 1.7-2 larger in M 33 than in M 31 . This shows that the magnetic field strength and/or the CRE density are higher on small scales in M 33. Due to the high SF activity in M 33, synchrotron, free-free and warm-dust emission are significant on small scales and show a fast decrease towards $a \simeq 3-4 \mathrm{kpc}$, after which an increase on larger scales occurs.

Figures 9-11 show the (total) radio-IR correlation, freefree-IR correlation and synchrotron-IR correlation versus spatial scale in M 31 and M 33. The radio-IR correlations (Fig. 9) (particularly RC-24/70 $\mu \mathrm{m}$ ) on $a<2.5 \mathrm{kpc}$ in M 31 are weaker than in M 33, without significant correlation $\left(r_{w}<0.5\right)$ on the smallest scale.

The free-free and IR emissions are well correlated $\left(r_{w}>0.5\right)$ on all scales in both galaxies (Fig. 10). On scales $a \leq 1.5 \mathrm{kpc}$, the correlations are stronger in M 33 than in M 31, depending on 

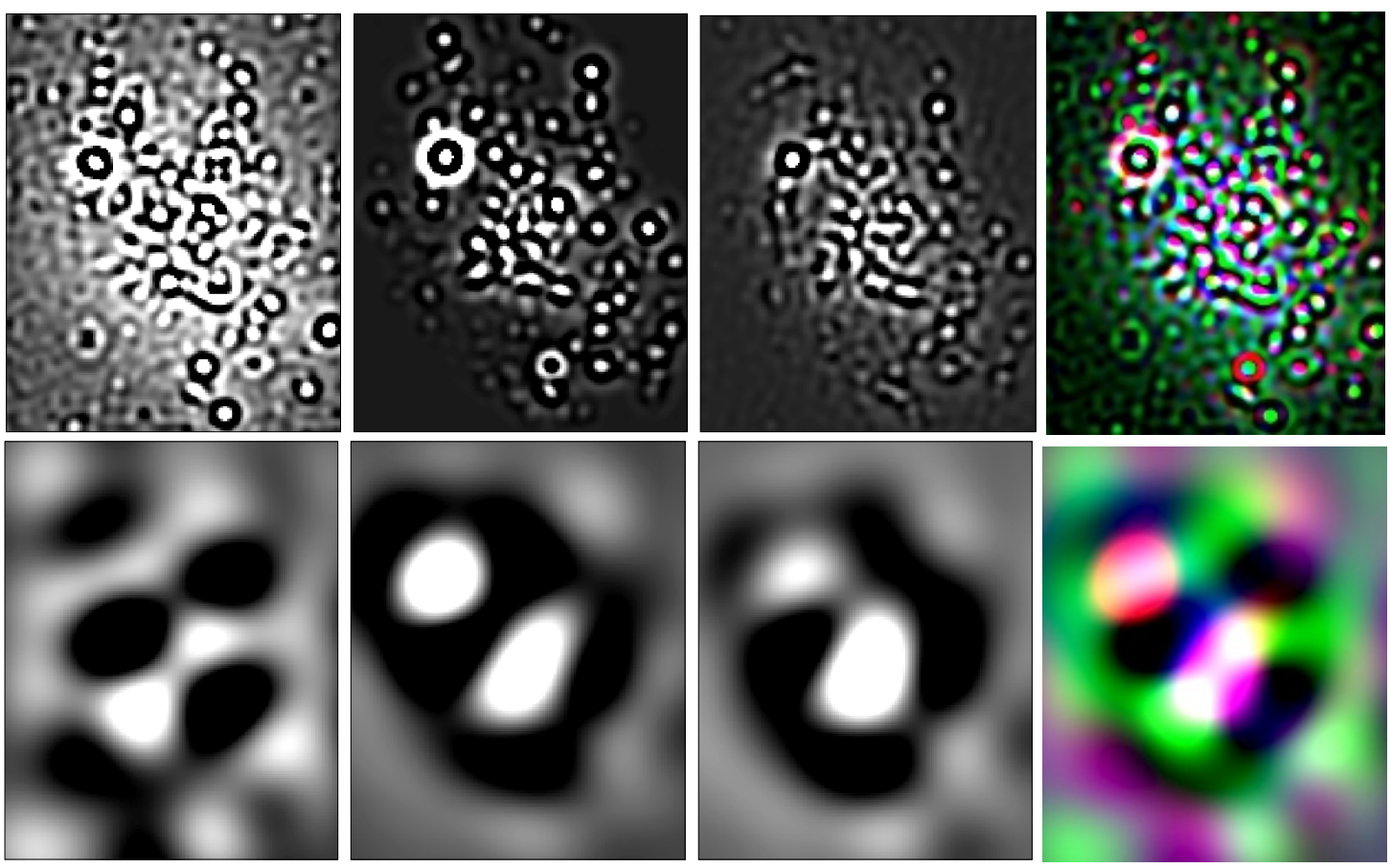

Fig. 7. M 33 maps of the synchrotron $(20 \mathrm{~cm}$, left), free-free $(20 \mathrm{~cm}$, middle), and $160 \mu \mathrm{m}$ (right) emission on the smallest scale of $a \sim 0.4 \mathrm{kpc}$ (upper row) and a large scale of $a=12.5^{\prime} \simeq 3 \mathrm{kpc}$ (bottom row) in arbitrary units. The map size is $42^{\prime} \times 54^{\prime}$. Also shown are the composite maps (red: free-free, green: synchrotron, and blue: $160 \mu \mathrm{m}$ emission).
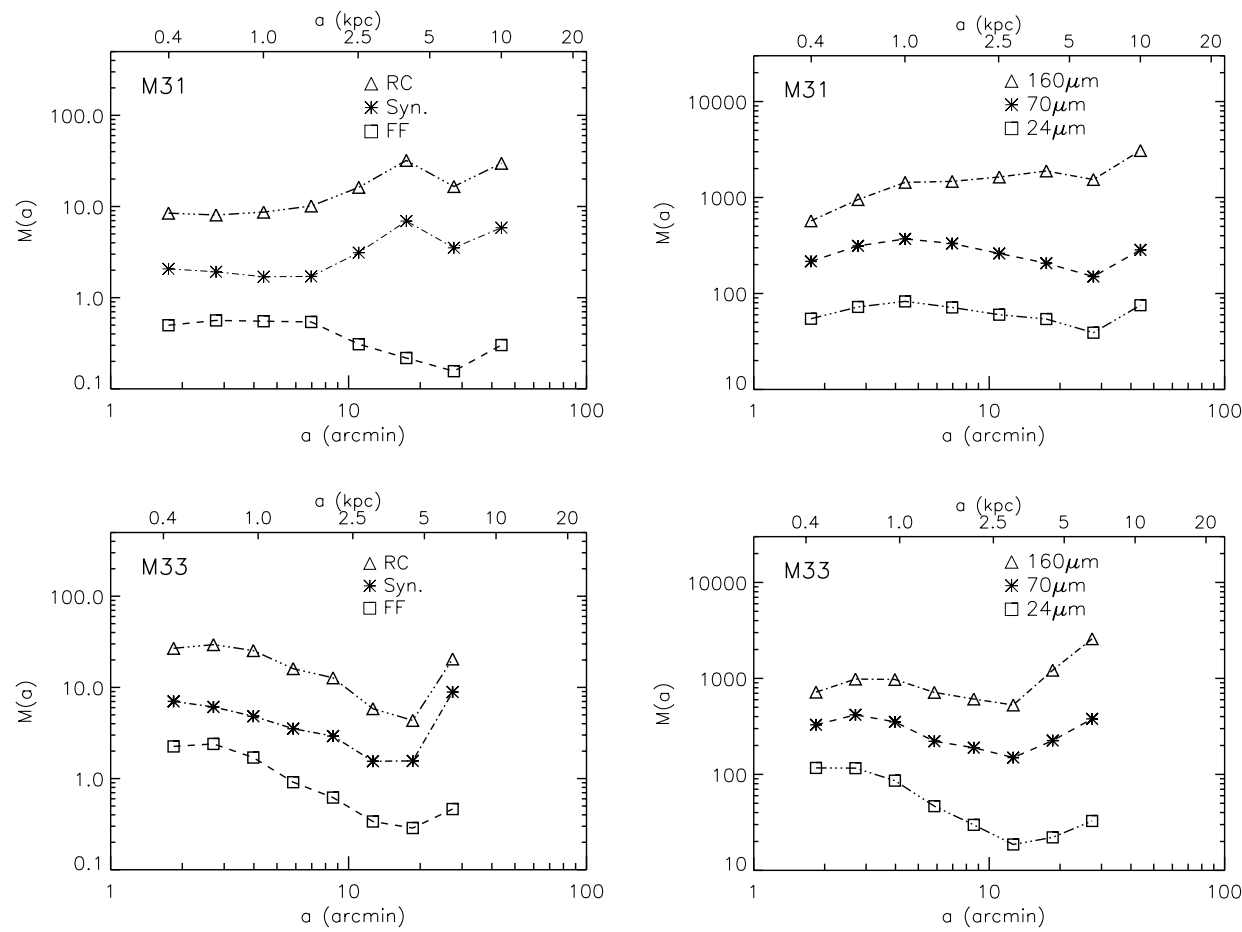

Fig. 8. Wavelet spectra of the observed $20 \mathrm{~cm}$ radio continuum (RC), synchrotron (Syn.), and free-free (FF) emissions (left) and IR emissions (right) from M 31 (top) and M 33 (bottom), shown in arbitrary units. The errors calculated by adding Gaussian noise (with an amplitude of one $\sigma$ noise level of the maps) before the decomposition are smaller than the symbols.

the IR wavelength. This indicates that the importance of young $\mathrm{O} / \mathrm{B}$ stars in heating the dust is higher in M 33 than in M 31. In this early-type galaxy, the dust is largely heated by ISRF (e.g. Hoernes et al. 1998), leading to a lower mean dust temperature (Tabatabaei \& Berkhuijsen 2010) and a weaker free-free-IR correlation than in M 33. The free-free emission is more tightly correlated with the $24 \mu \mathrm{m}$ than the $70 \mu \mathrm{m}$ emission in $\mathrm{M} 33$, as expected, but it is the other way around in M 31. This, along with the observed excess in the $24 \mu \mathrm{m}$-to- $70 \mu \mathrm{m}$ intensity ratio, indicates that sources other than interstellar dust heated by massive stars contribute to the $24 \mu \mathrm{m}$ emission in M 31 (see Tabatabaei \& Berkhuijsen 2010). 

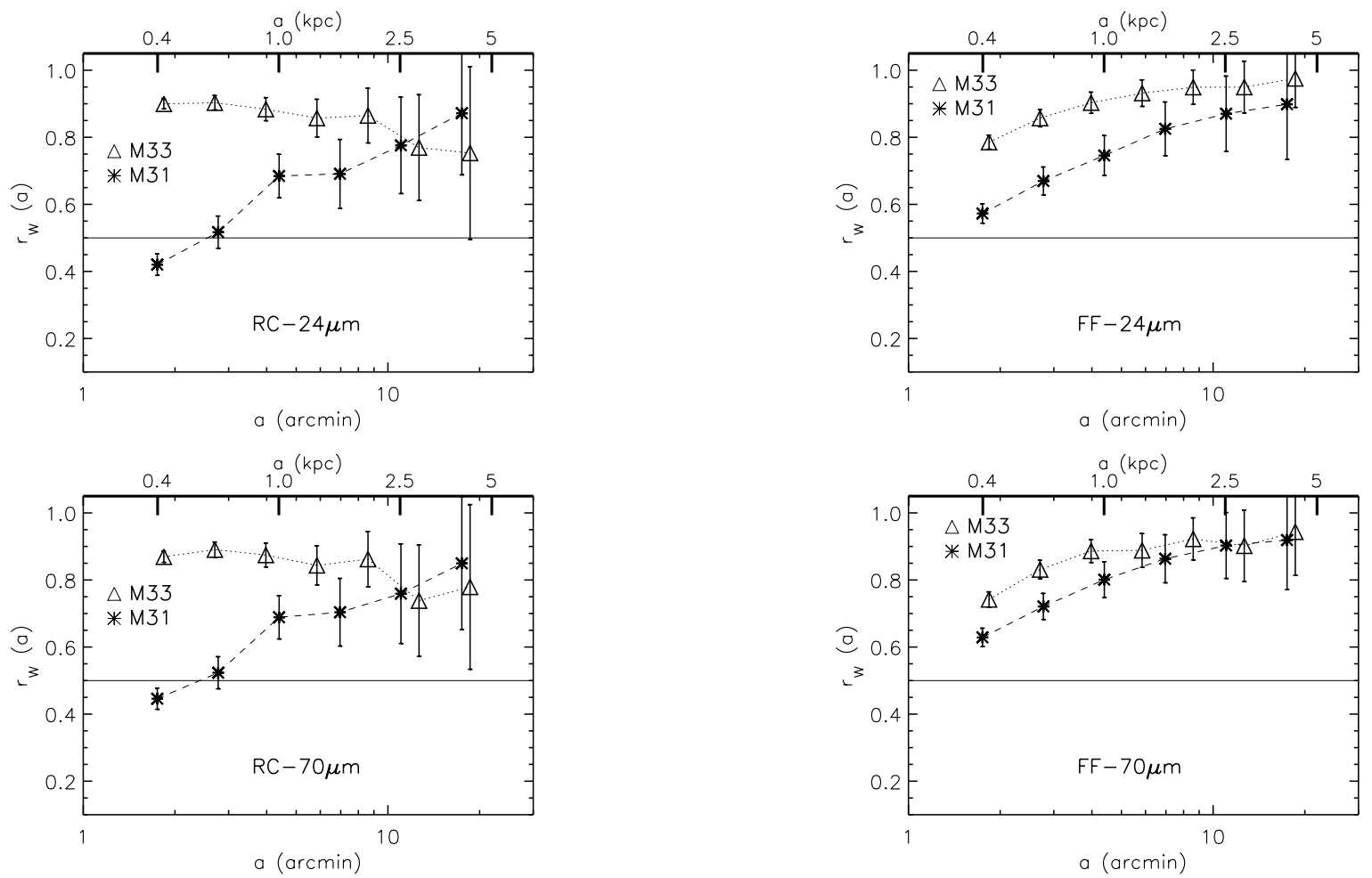

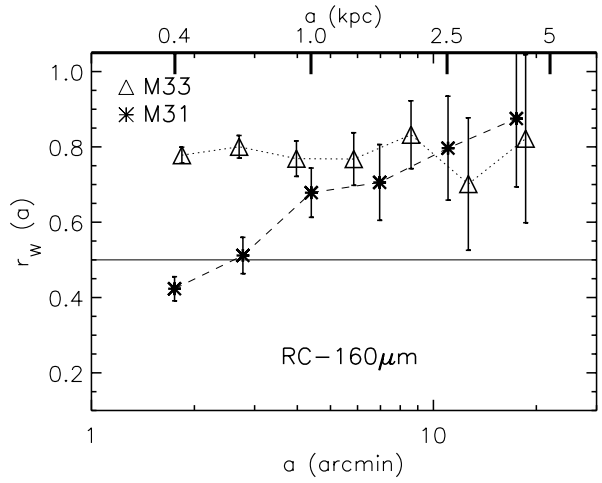

Fig. 9. Scale-by-scale cross-correlations between IR and observed $20 \mathrm{~cm}$ radio continuum (RC) emission from M 31 and M 33 .

The synchrotron-IR correlations in M 31 and M 33 (Fig. 11) are quite different. In M 31, the correlations are good $\left(r_{w}>0.7\right)$ on $a>2 \mathrm{kpc}$, but decrease towards smaller scales. On $a<1 \mathrm{kpc}$, no significant correlation $\left(r_{w}<0.5\right)$ exists. In M 33, however, the synchrotron-IR correlation reasonably holds $\left(0.5<r_{w}<0.7\right)$ on scales $a<2 \mathrm{kpc}$, while it is weak on scales of 3-4 kpc due to the different distributions of synchrotron and IR emission on these scales (see Fig. 7, bottom).

\section{Discussion}

\subsection{CRE propagation length and magnetic field structure in M 31 and $M 33$}

On small scales $(a<1 \mathrm{kpc})$, the synchrotron-IR correlation is stronger in M 33 than in M 31, but on large scales $(a>2.5 \mathrm{kpc})$ it is stronger in M 31 (Fig. 11). To understand these differences, we look at the properties of the magnetic field and CREs in $\mathrm{M} 31$ and $\mathrm{M} 33$, because the synchrotron emission is proportional to both the strength of the total magnetic field $\left(B_{\text {tot }}\right)$ and the number of CREs. $B_{\text {tot }}$ consists of turbulent $\left(B_{\mathrm{tur}}\right)$ and ordered $\left(B_{\text {ord }}\right)$ magnetic fields $\left(B_{\text {tot }}^{2}=B_{\text {tur }}^{2}+B_{\text {ord }}^{2}\right)$. Since $B_{\text {tur }}$ and the number of young CREs are enhanced in SF regions, i.e. on small scales, the synchrotron emission is also increased (Chyży 2008; Krause 2009), while the large-scale field $B_{\text {ord }}$ is not enhanced in SF regions (Frick et al. 2001; Chyży 2008). Hence one may speculate that a higher SFR per unit area $\left(\Sigma_{\mathrm{SFR}}\right)$ like in M 33 (see Table 3) improves the synchrotron-IR correlation on small scales. However, Boulanger \& Perault (1988) did not find a correlation between radio and IR emission for the starforming Orion nebula of a few 100 pc. As the Milky Way also has a higher $\Sigma_{\text {SFR }}$ than M 31, this is inconsistent with the above speculation.

The differences between the correlations in M 31 and M 33 are better explained by differences in the propagation of CREs. On our smallest scale of $a=0.4 \mathrm{kpc}$, the reasonable synchrotron-IR correlation in M 33 implies that the structures of the two intensity maps are similar and that the positions of maxima and minima on the sky largely agree. This is visible in Fig. 6 (bottom), where more or less the same bright sources (which are complexes of SF regions) can be traced in the synchrotron and the $160 \mu \mathrm{m}$ maps. This means that CREs are still 

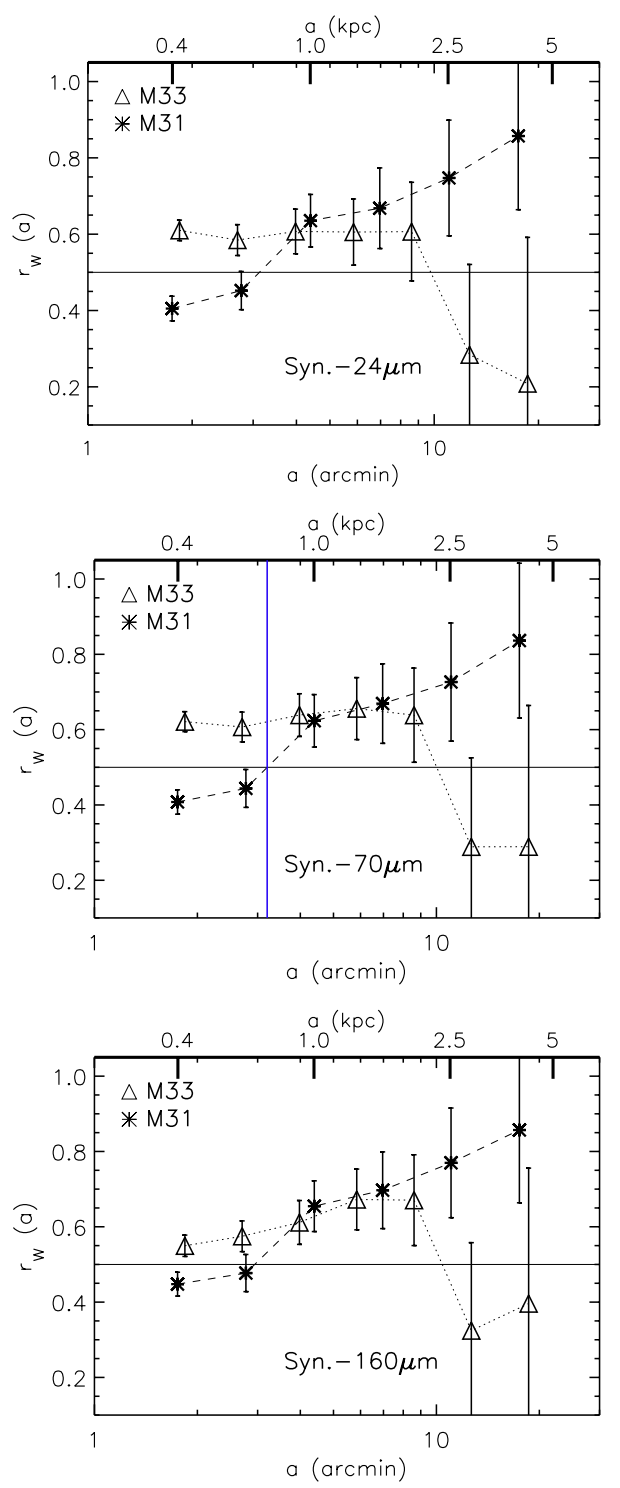

Fig. 11. Scale-by-scale cross-correlations between IR and $20 \mathrm{~cm}$ synchrotron emission from M 31 and M 33. The vertical line in the middle panel indicates $a=l_{\text {dif }}$ for M 31 .

near their places of origin in the SF regions. Thus, the propagation length of CREs $\left(l_{\text {prop }}\right)$ in M 33 is smaller than $0.4 \mathrm{kpc}$. In M 31, however, the insignificant correlation $\left(r_{w}<0.5\right)$ on $a<1 \mathrm{kpc}$ implies that the structures of synchrotron and dust emission differ. Figure 6 (top) shows this difference: the dust emission mainly emerges from the dust lanes and SF regions in the spiral arms, whereas the synchrotron emission comes from the bright diffuse ring. This indicates that the bulk of the CREs have propagated away from their places of origin in the SF regions. Since the synchrotron-IR correlation holds $\left(r_{w} \geq 0.5\right)$ on scales of $a \gtrsim 1 \mathrm{kpc}$, we conclude that the CREs must have propagated over a length of $l_{\text {prop }} \simeq 1 \mathrm{kpc}^{7}$. Hence, $l_{\text {prop }}$ in M 31 is larger than in M 33. Possible reasons for this difference are discussed below.

The synchrotron-IR correlation on large scales $(a>2.5 \mathrm{kpc})$ is due to diffuse emission associated with the " $10 \mathrm{kpc}$ ring" in M 31 and with the central region in M 33. Because $l_{\text {prop }}$ in

7 The kpc scales used here refer to the major axis.
M 31 is larger than in M 33, the synchrotron emission will be more diffuse, leading to a better correlation with the diffuse dust emission in M 31. The bright HII region complex NGC 604 and the central disk seen in the dust emission from M 33 on scales 3-4 kpc (e.g. see Fig. 7) have no counterpart in the synchrotron emission, which causes the low correlation coefficients on $a=3-4 \mathrm{kpc}$. This could be due to the combined effect of the small $l_{\text {prop }}$ in the disk that keeps the CREs near SF regions, and the fact that the large-scale $B_{\text {tot }}$ is not confined to the thin disk in M 33. Tabatabaei et al. (2008) found that M 33 has a vertical magnetic field extending to $R \approx 5 \mathrm{kpc}$, which is strongest in the central disk. This causes propagation of CREs from the SF regions in the thin disk into the halo, as observed in edge-on galaxies (e.g. Heesen et al. 2009). CRE propagation along the vertical field into the halo reduces the synchrotron intensity in the SF regions (see also Tabatabaei et al. 2007c). This further weakens the synchrotron-IR correlation because the dust emission is concentrated in the thin disk.

In M 31, since $B_{\text {tot }}$ is confined to the dusty thin disk (no synchrotron halo, Moss et al. 1998; Fletcher et al. 2004), the better correlations on large scales are due to the similar structures in the thin disks (as shown in Fig. 6). This suggests coupling of the magnetic field to the gas mixed with the dust, which could explain the synchrotron-IR correlation in M 31, as proposed by Hoernes et al. (1998).

\subsection{CRE propagation models}

As indicated above, the difference in $l_{\text {prop }}$ between M 31 and M 33 could be related to their different magnetic field properties. The degree of order of the magnetic field plays an important role in the propagation of CREs, both in models of streaming instability (e.g. Kulsrud 2005; Enßlin et al. 2011) and in diffusion models (e.g. Chuvilgin \& Ptuskin 1993; Breitschwerdt et al. 2002; Dogiel \& Breitschwerdt 2012; Shalchi 2009). A larger ordering, i.e. a larger $B_{\text {ord }} / B_{\text {tur }}$, helps CREs to propagate over larger distances along the ordered magnetic field resulting in a larger $l_{\text {prop }}$. This is in agreement with the observational results: $B_{\text {ord }} / B_{\text {tur }}$ is indeed larger in M 31 than in M 33 (see Table 3), as the larger $l_{\text {prop }}$ suggests. This offers a new way to estimate $l_{\text {prop }}$ from the synchrotron-IR correlation in galaxies.

In the case of streaming, CREs move along the lines of the ordered field with the Alfvén velocity $\left(v_{\mathrm{A}}\right)$ and propagate over a distance $l_{\text {prop }}=v_{\mathrm{A}} t_{\mathrm{CRE}}$ during their lifetime $\left(t_{\mathrm{CRE}}\right)$. Observations indicate equipartition between the kinetic and magnetic energy densities, so that $v_{\mathrm{A}} \approx$ const. If the CRE lifetime is limited by synchrotron loss, $t_{\mathrm{CRE}}=t_{\mathrm{syn}} \propto B_{\text {tot }}^{-1.5} v^{-0.5}$, where $v$ is the frequency at which the CREs of energy $E$ radiate ${ }^{8}$ (neglecting inverse Compton losses, which are smaller than synchrotron losses; Murphy 2009), we get $l_{\text {prop }} \propto B_{\text {tot }}^{-1.5} v^{-0.5}$. If $t_{\text {CRE }}$ is given by the time that the CREs reside in the thin galactic disk until they move into the thick disk/halo, the confinement time $\left(t_{\text {conf }}\right)$, then $l_{\text {prop }} \propto t_{\text {conf }}$.

However, as streaming needs longer stretches of the ordered field to be effective, it may not be important in the turbulent thin disk of galaxies (Dogiel \& Breitschwerdt 2012; Enßlin et al. 2011). Furthermore, there is evidence that in the Milky Way on scales $<1 \mathrm{kpc}$ CRE propagation is isotropic (Strong et al. 2007; Stepanov et al. 2012), which is inconsistent with streaming. In the following, we assume that generally CRE diffusion dominates, except in regions with highly ordered fields, where streaming may dominate. 
Table 3. Magnetic field strengths, $\Sigma_{\mathrm{SFR}}$, and $l_{\mathrm{dif}}$ in 5 nearby galaxies.

\begin{tabular}{lcccccc}
\hline \hline Galaxy & $\begin{array}{c}B_{\text {tot }} \\
(\mu \mathrm{G})\end{array}$ & $\begin{array}{c}B_{\text {ord }} \\
(\mu \mathrm{G})\end{array}$ & $\begin{array}{c}B_{\text {tur }} \\
(\mu \mathrm{G})\end{array}$ & $B_{\text {ord }} / B_{\text {tur }}$ & $\begin{array}{c}\Sigma_{\text {SFR }} \\
\left(M_{\odot} \mathrm{Gyr}^{-1} \mathrm{pc}^{-2}\right)\end{array}$ & $\begin{array}{c}l_{\text {dif }} \\
(\mathrm{pc})\end{array}$ \\
\hline M 31 & $6.6 \pm 0.3$ & $4.3 \pm 0.3$ & $5.0 \pm 0.2$ & $0.86 \pm 0.07$ & $0.6 \pm 0.1$ & $730 \pm 90$ \\
M 33 & $8.1 \pm 0.5$ & $2.8 \pm 0.3$ & $7.6 \pm 0.5$ & $0.37 \pm 0.05$ & $3.0 \pm 0.6$ & $<400$ \\
NGC 6946 & $16.0 \pm 1.5$ & $9.9 \pm 2.1$ & $12.6 \pm 2.7$ & $0.79 \pm 0.24$ & $7.1 \pm 1.8$ & $1700 \pm 200^{1}$ \\
LMC & 4.2 & $1.1^{2}$ & $4.1^{2}$ & $0.27 \pm 0.10$ & $2.6^{3}$ & $200 \pm 90^{4}$ \\
M 51 & 19.7 & $11.5^{5}$ & $16.0^{5}$ & $0.72 \pm 0.26$ & $12^{6}$ & $>500^{7}$ \\
\hline
\end{tabular}

Notes. Note that apart from $l_{\text {dif }}$ all values refer to $30^{\prime}<R<50^{\prime}$ in M 31, $R<21^{\prime}$ in M 33, $R<3.9^{\prime}$ in M 51 , and $R<3.8^{\circ}$ in the LMC. The 30 Dor region and the star-forming ridge are not considered for the LMC. For M51, $l_{\text {dif }}$ is determined from the total radio continuum-IR correlation (see text).

References. 1) Tabatabaei et al. (2013); 2) Gaensler et al. (2005); 3) Whitney et al. (2008), revised for a Salpeter IMF; 4) Hughes et al. (2006) and (in prep.); 5) Fletcher et al. (2011); 6) Leroy et al. (2008); 7) Dumas et al. (2011).

In the case of diffusion, the particles are scattered by the irregularities in the turbulent magnetic field. The diffusion length $\left(l_{\text {dif }}\right)$ depends on the diffusion coefficient $(D)$ and $t_{\mathrm{CRE}}$ as $l_{\mathrm{dif}}=$ $\left(D t_{\mathrm{CRE}}\right)^{0.5}$, where $D \propto D_{\mathrm{E}} E^{0.5} \propto D_{\mathrm{E}} v^{0.25} B_{\text {tot }}^{-0.25}$, with $D_{\mathrm{E}}$ being the energy dependent diffusion coefficient. Models of diffusive CR propagation in the ISM indicate that $D_{\mathrm{E}} \propto\left(B_{\text {ord }} / B_{\text {tur }}\right)^{2}$ (Chuvilgin \& Ptuskin 1993; Breitschwerdt et al. 2002; Yan \& Lazarian 2004; Dogiel \& Breitschwerdt 2012; Shalchi 2009). Assuming that diffusion along $B_{\text {ord }}$ dominates, the parallel diffusion coefficient is $D_{\|} \propto\left(B_{\text {ord }} / B_{\text {tur }}\right)^{2} B_{\text {ord }}^{-1 / 3}$ (Shalchi 2009, Eq. (3.41)). Hence, observing at a fixed frequency, we expect that during their synchrotron lifetime $t_{\text {syn }}$ the CRE will diffuse over the pathlength:

$l_{\text {dif }} \propto\left(B_{\text {ord }} / B_{\text {tur }}\right) B_{\text {ord }}^{-1 / 6} B_{\text {tot }}^{-7 / 8}$,

where we used $t_{\text {syn }} \propto B_{\text {tot }}^{-1.5}$.

If, on the other hand, the period that the CREs spend in the galactic disk is the confinement time, then:

$l_{\text {dif }} \propto\left(B_{\text {ord }} / B_{\text {tur }}\right) B_{\text {ord }}^{-1 / 6} B_{\text {tot }}^{-1 / 8} t_{\text {conf }}^{1 / 2}$.

For M 31 we estimate $l_{\text {dif }}=730 \pm 90 \mathrm{pc}$, where $l_{\text {dif }}$ is the smallest scale on which the synchrotron-IR correlation is found to hold $\left(r_{w}=0.5\right)^{9}$. The smallest scales contain the SF regions, where most CREs are young (as suggested by Murphy et al. 2006), and $B_{\text {tot }}$ is larger than average $B_{\text {tot }}$. As the synchrotron emission from the smallest scale in M 33 is about 1.9 times that in M 31 (Sect. 4.1), we estimate that $B_{\text {tot }}$ is about $(1.9)^{1 /\left(1+\alpha_{n}\right)} \simeq 1.5$ times stronger (assuming equipartition between the magnetic field and CREs energy densities and using a nonthermal spectral index of $\alpha_{n} \simeq 0.6$, Tabatabaei et al. 2007c). With the smaller value of $B_{\text {ord }} / B_{\text {tur }}$ and $B_{\text {ord }}$ (see Table 3 ), and equal $t_{\text {conf }}$ (see Sect. 5.3), this leads to $l_{\text {dif }}=320 \pm 70$ pc in M 33, in agreement with our estimate of $<400 \mathrm{pc}$ from the synchrotron-IR correlation. For comparison, using the somewhat lower mean value of $B_{\text {tot }}$ given in Table 3 yields $l_{\text {dif }}=330 \pm 70$ pc.

So far we have measured $l_{\text {dif }}$ in the sky plane, i.e. along the major axis of the galaxy and perpendicular to it. We may estimate $l_{\text {dif }}$ in the disk plane by assuming that the diffusion lengths along the major axis $\left(l_{x}\right)$ and the minor axis $\left(l_{y}\right)$ are the same, as is indicated by the observed isotropy of CREs in the Milky Way on scales <1 kpc (Strong et al. 2007; Stepanov et al. 2012). The mean diffusion length along the plane then is $l_{\mathrm{dif}, x y}=\sqrt{l_{x}^{2}+l_{y}^{2}}=l_{\mathrm{dif}} \sqrt{2}$.

9 This is determined using the synchrotron- $70 \mu \mathrm{m}$ correlation, as in M 31 the $70 \mu \mathrm{m}$ emission traces SF and CREs sources better than other IR bands (Sect. 4.1).

\subsection{Comparison with other galaxies}

NGC 6946 and the LMC are the only two nearby galaxies, for which similar scale-by-scale studies of the synchrotron-IR correlation exist (Tabatabaei et al. 2013; Hughes et al., in prep.), and it is interesting to compare the five galaxies. Table 3 shows all values of $l_{\text {dif }}$, the smallest scale on which the synchrotron and IR emission are correlated. For M 51, Dumas et al. (2011) presented the radio continuum-IR correlation as a function of scale. The smallest scale on which the radio continuum (including both synchrotron and free-free emission) and IR emission are correlated is a lower limit for $l_{\text {dif }}$ (also given in Table 3 ), because the good free-free-IR correlation increases the radio-IR correlation on small scales.

We first tested if $l_{\text {dif }}$ indeed follows Eq. (12) given above, but did not find such a correlation, especially not an inverse dependence on $B_{\text {tot }}$. This indicates that the synchrotron lifetime $t_{\text {syn }}$ does not determine the CRE lifetime because the confinement time $t_{\text {conf }}$ is shorter than $t_{\text {syn }}$, which was also found by Lacki et al. (2010). Interestingly, we do find a clear dependence on $\left(B_{\text {ord }} / B_{\text {tur }}\right.$ ) (see Fig. 12a), which is consistent with Eq. $(13)^{10}$. The fact that this dependency exists shows that the confinement time in the five galaxies will be approximately the same. This is understandable because the size of star formation regions and the scale height of the thin disk (typically $0.3 \mathrm{kpc}$, Krause 2009) are about the same in galaxies, so the CREs need about the same time to diffuse out of the disk.

NGC 6946 has "magnetic arms" where the magnetic field is very regular (Beck 2007; Tabatabaei et al. 2013). The fact that the point for NGC 6946 in Fig. 12a is above the dotted line may indicate that along these arms CRE streaming occurs, while diffusion dominates elsewhere. It is also possible that $t_{\text {conf }}$ in NGC 6946 is very long because CREs in the magnetic arms will be more strongly confined to the thin disk than in the spiral arms. A longer $t_{\text {conf }}$ is consistent with the conclusion of Tabatabaei et al. (2013) that $l_{\text {dif }}$ in NGC 6946 agrees with the diffusion length expected from synchrotron and Inverse Compton losses during $t_{\text {syn }}\left(t_{\text {conf }} \sim t_{\text {syn }}\right)$.

Since between the galaxies the values of $B_{\text {tur }}$ vary less than those of $B_{\text {ord }}$, Eq. (13) may be approximated by $l_{\text {dif }} \propto B_{\text {ord }}^{5 / 6}$. Figure $12 \mathrm{~b}$ shows that this is also consistent with our data.

We note that $l_{\text {dif }}$ may also depend on the star-formation rate (SFR) per unit area $\left(\Sigma_{\mathrm{SFR}}\right)$. In galaxies with high $\Sigma_{\mathrm{SFR}}$ the filling

10 The correlation with the full Eq. (13) is somewhat worse than that with $\left(B_{\text {ord }} / B_{\text {tur }}\right)$ alone, but still consistent with the data. The extra factors $B_{\text {ord }}^{-1 / 6}$ and $B_{\text {tot }}^{-1 / 8}$ apparently worsen the correlation with $B_{\text {ord }} / B_{\text {tur }}$. 

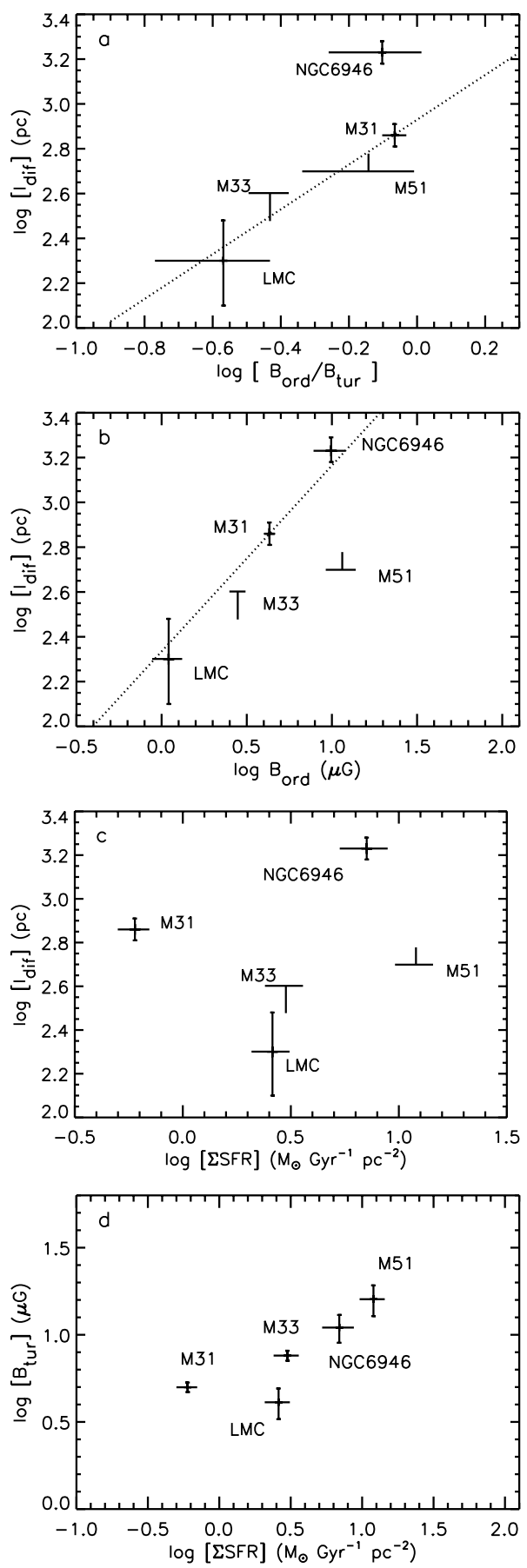

Fig. 12. Smallest scale on which the $R C$ and IR emissions are correlated. From top to bottom $l_{\text {dif }}$ versus $B_{\text {ord }} / B_{\text {tur }}$ a), $B_{\text {ord }} \mathbf{b}$ ), and $\Sigma_{\mathrm{SFR}} \mathbf{c}$ ). Also shown is $B_{\text {tur }}$ versus $\Sigma_{\mathrm{SFR}} \mathbf{d}$ ). For the LMC and M 51, $20 \%$ uncertainty is assumed. The dotted lines in a) and b) are not fits, but show the relation expected from Eq. (13) with slope $=1$ in a) and slope $=5 / 6$ in b), adjusted to M 31 .

factor of young CREs on small scales (i.e. in SF regions) will be higher than in galaxies with low $\Sigma_{\mathrm{SFR}}$, which could lead to a smaller $l_{\text {dif }}$ than that of the bulk of CREs. Breitschwerdt et al. (2002) showed that a higher $\Sigma_{\text {SFR }}$ favors convective propagation of CREs from the SF regions into the halo, which reduces the time that CREs spend in the thin disk and will decrease $l_{\text {dif }}$. Furthermore, $B_{\text {tur }}$ increases in SF regions and could lead to a lower $l_{\text {dif }}$. Figure $12 \mathrm{~d}$ shows that $B_{\text {tur }}$ is indeed correlated with $\Sigma_{\text {SFR }}$ (see also Chyży 2008; Krause 2009; Tabatabaei et al. 2013), but $l_{\text {dif }}$ is not (Fig. 12c). Instead, $l_{\text {dif }}$ scales with the ratio $B_{\text {ord }} / B_{\text {tur. }}$. The reason for the lack of correlation between $l_{\text {dif }}$ and $\Sigma_{\text {SFR }}$ could be the weak dependence of $B_{\text {tur }}$ on $\Sigma_{\text {SFR }}$ (with an exponent of about 0.2 within galaxies, Chyży 2008; Tabatabaei et al. 2013, and about 0.6 between galaxies, see Fig. 12d). Moreover, $B_{\text {ord }}$ is not related to the SFR (Frick et al. 2001; Chyży 2008; Krause 2009; Tabatabaei et al. 2013), but is controlled by various dynamical and environmental effects (Fletcher et al. 2011). Thus it is expected that $l_{\text {dif }}$ does not directly scale with $\Sigma_{\mathrm{SFR}}$. Hence, although the presence of massive star formation influences the field structure, it is the ratio $B_{\text {ord }} / B_{\text {tur }}$ that is crucial for the propagation of the CREs.

\subsection{Comparison with smoothing experiments}

Murphy et al. (2008) determined propagation lengths of CREs in 18 well-resolved galaxies ( $<1 \mathrm{kpc}$ resolution) by smoothing $70 \mu \mathrm{m}$ Spitzer images until they matched the $22 \mathrm{~cm}$ Westerbork maps, corrected for thermal emission. Using a wavelet technique, they separated the $70 \mu \mathrm{m}$ images in a "structure component" of scales $<1 \mathrm{kpc}$ containing the star formation regions and a diffuse "disk component" of scales $>1 \mathrm{kpc}$, heated by older stars. By separately smoothing these components with exponential kernels, until their sum matched the corrected radio image, they determined a structure scale length and a disk scale length, which they interpreted as diffusion lengths of two populations of CREs. There are some major differences between the approach of Murphy et al. (2008) and our work, making a comparison of results difficult.

1. Murphy et al. (2008) assume that supernova remnants (SNRs) located in the structure component as well as reacceleration in ISM shock waves in the disk component are sources of CREs. However, re-acceleration of CREs in the ISM has not been proven to exist - neither theoretically nor observationally. In our work, we assume that all CREs originate from SNRs.

2. Murphy et al. (2008) claim that the CREs in the structure component have been accelerated only recently and have a typical age of $\gtrsim 4$ times shorter than the CREs in the disk component. In our work, we assume that that CRE acceleration time is much shorter than the CRE radiation time and we argue that the radiation time is limited by the confinement time in the thin disk.

3. Murphy et al. (2008) ignore the role of the magnetic fields in diffusion of CREs. Their calculations are based on some assumed values of the total magnetic field strength. In our work, we show the importance of the magnetic fields with respect to strength and ordering. We use the magnetic field strengths independently measured for each galaxy.

4. Both the structure and disk scale lengths derived by Murphy et al. (2008) decrease with increasing $\Sigma_{\mathrm{SFR}}$, especially for galaxies with high $\Sigma_{\mathrm{SFR}}$. They attribute it to the increasing importance of the structure component that contains the $\mathrm{SF}$ regions. However, our data do not show a dependence of $l_{\text {dif }}$ on $\Sigma_{\text {SFR }}$ (see Fig. 12, lower middle panel), although our range of $\Sigma_{\mathrm{SFR}}$ is larger than that of the sample of Murphy et al. (2008).

Our main concern about the work of Murphy et al. (2008) is the reality of the disk component as a CRE source distribution. We believe that their scale $l_{\text {disk }}$ is the difference between the exponential radial scale lengths of the diffuse dust emission powered by old stars and the extended synchrotron emission (see their example for NGC 6946). Since old stars are not progenitors of SNRs, they cannot produce CREs. Moreover, if there really 
would be two types of CRE sources, this should cause two peaks in the wavelet cross-correlation function, one at small and one at large scales. Something like that may be visible in NGC 6946 (Tabatabaei et al. 2013), but clearly not in M 31 and M 33. It seems unlikely that the "diffuse source component" exists.

Murphy et al. (2008) also derived global scale lengths, $l_{\text {glob }}$, after smoothing of the unseparated, observed $70 \mu \mathrm{m}$ image, and it is interesting to compare their values for M 51 and NGC 6946 with our $l_{\text {dif. }}$. For M 51, they find $l_{\text {glob }}=500 \pm 100 \mathrm{pc}$ which is consistent with our lower limit of $l_{\text {dif }}>500$ pc. For NGC 6946, however, they derive $l_{\text {glob }}=600 \pm 140 \mathrm{pc}$, much lower than our $l_{\text {dif }}=1700 \pm 200$ pc. Recently, Murphy et al (2012) applied the smoothing method to the LMC $\left(R<2.7^{\prime}\right)$ and found a propagation length of $200-400 \mathrm{pc}$, depending on the smoothing kernel used. This agrees with the value for the LMC (without 30 Dor) of $l_{\text {dif }}=200 \pm 90 \mathrm{pc}$ in Table 3 (Hughes et al., in prep.). However, as there is no general consistency between the results of the two methods, a thorough comparison for a sample of galaxies with low and high $\Sigma_{\mathrm{SFR}}$, based on the same data, will be needed to better understand the differences.

\section{Summary and conclusion}

Separating the $20 \mathrm{~cm}$ free-free and synchrotron emission, we find bright synchrotron emission close to the sites of star formation, concentrated in a smooth wide ring in M 31 and in a smooth spiral pattern in M 33. On the resolved scale of star forming regions, $\simeq 200 \mathrm{pc}$, both the free-free emission and the synchrotron emission are stronger in $M 33$ than in M 31. The wavelet analysis shows that in M 31 and M 33 the free-free emission is similarly distributed versus scale (i.e. dominant on small scales, $a \lesssim 2 \mathrm{kpc}$ ), while the synchrotron emission behaves differently: it is dominant on large scales $(\simeq 4 \mathrm{kpc})$ in M 31, unlike in M 33.

Using the Spitzer-MIPS data at 24, 70, and $160 \mu \mathrm{m}$, we derive radio-IR correlations as a function of scale, separately for free-free and synchrotron emission. The free-free and IR emissions are correlated on all scales studied $(0.4 \leq a \leq 10 \mathrm{kpc})$ in both M 33 and M 31. The synchrotron-IR correlations, however, are different in M 31 and M 33, on small scales ( $<1 \mathrm{kpc})$ as well as on larger scales. In M 31 only little correlation exists on scales $<1 \mathrm{kpc}$, but in M 33 the synchrotron-IR correlation is reasonable on small scales. We propose that the differences can be explained by the difference in diffusion length of CREs caused by the different magnetic field properties of M 31 and M 33.

Combining our results with data on NGC 6946, LMC and M 51, we find evidence that the smallest scale, on which the synchrotron-IR correlation exists, depends on the ratio of the ordered-to-turbulent magnetic field strength. We show that this is consistent with diffusion of CREs in the disk until they escape into the halo. The confinement times of the CREs in the disks must be about the same. In our sample, no correlation exists between $l_{\text {dif }}$ and $\Sigma_{\mathrm{SFR}}$.

Our results show the fundamental importance of CRE propagation and magnetic field structure for the radio-IR correlation within a galaxy, and offer a new method to measure the diffusion length $l_{\text {dif }}$ of CREs in galaxies.

\section{Outlook}

Whether the propagation length of CREs in galaxies indeed increases with increasing $B_{\text {ord }} / B_{\text {tur }}$, as our results suggest, should be determined from the synchrotron-IR cross-correlation as a function of scale for a large number of galaxies with different degrees of field ordering and field strengths. For each galaxy a realistic separation of free-free and synchrotron emission is required (Tabatabaei et al. 2007c), like we did for M 31 and M 33 (see Sect. 3).

Future observations should be performed at different frequencies. At frequencies lower than $1 \mathrm{GHz}$ the propagation length of CREs should not change much if the CRE confinement time is independent of CRE energy. On the other hand, the lifetime of CREs in galaxies radiating at frequencies higher than about $5 \mathrm{GHz}$ is expected to be dominated by synchrotron loss, so that the dependence of propagation length on the field strength changes from Eq. (13) to Eq. (12). The difference between the propagation lengths at two widely separated frequencies will allow us to measure the diffusion coefficient and to provide constraints for models of cosmic-ray propagation.

Acknowledgements. F.S.T. acknowledges the support by the DFG via the grant TA 801/1-1. R.B. acknowledges the support by DFG FOR1254. We thank Annie Hughes, Brent Groves, and the anonymous referee for critical and helpful comments. Discussions with Andreas Shalchi, Gianfranco Brunetti and Torsten Enßlin on CRE diffusion improved our insight into this process.

\section{References}

Aurell, E., Frick, P., \& Shaidurov, V. 1994, Phys. D Nonlinear Phenomena, 72, 95

Beck, R. 2007, A\&A, 470, 539

Beck, R., \& Golla, G. 1988, A\&A, 191, L9

Beck, R., Berkhuijsen, E. M., \& Hoernes, P. 1998, A\&AS, 129, 329

Bell, E. F. 2003, ApJ, 586, 794

Berkhuijsen, E. M., \& Humphreys, R. M. 1989, A\&A, 214, 68

Blair, W. P., Kirshner, R. P., \& Chevalier, R. A. 1982, ApJ, 254, 50

Block, D. L., Bournaud, F., Combes, F., et al. 2006, Nature, 443, 832

Boulanger, F., \& Perault, M. 1988, ApJ, 330, 964

Breitschwerdt, D., Dogiel, V. A., \& Völk, H. J. 2002, A\&A, 385, 216

Chuvilgin, L. G., \& Ptuskin, V. S. 1993, A\&A, 279, 278

Chyży, K. T. 2008, A\&A, 482, 755

Condon, J. J. 1992, ARA\&A, 30, 575

de Jong, T., Klein, U., Wielebinski, R., \& Wunderlich, E. 1985, A\&A, 147, L6

Dennefeld, M., \& Kunth, D. 1981, AJ, 86, 989

Devereux, N. A., Price, R., Wells, L. A., \& Duric, N. 1994, AJ, 108, 1667

Dickinson, C., Davies, R. D., \& Davis, R. J. 2003, MNRAS, 341, 369

Dogiel, V., \& Breitschwerdt, D. 2012, EAS Pub. Ser., 56, 61

Dumas, G., Schinnerer, E., Tabatabaei, F. S., et al. 2011, AJ, 141, 41

Edwards, A. L. 1979, Multiple Regression and Analysis of Variance and Covariance (San Francisco: W.H. Freeman and Company)

Enßlin, T., Pfrommer, C., Miniati, F., \& Subramanian, K. 2011, A\&A, 527, A99

Fletcher, A., Berkhuijsen, E. M., Beck, R., \& Shukurov, A. 2004, A\&A, 414, 53

Fletcher, A., Beck, R., Shukurov, A., Berkhuijsen, E. M., \& Horellou, C. 2011, MNRAS, 412, 2396

Freedman, W. L., Wilson, C. D., \& Madore, B. F. 1991, ApJ, 372, 455

Frick, P., Beck, R., Berkhuijsen, E. M., \& Patrickeyev, I. 2001, MNRAS, 327, 1145

Gaensler, B. M., Haverkorn, M., Staveley-Smith, L., et al. 2005, Science, 307, 1610

Gavazzi, G., Cocito, A., \& Vettolani, G. 1986, ApJ, 305, L15

Gordon, K. D., Pérez-González, P. G., Misselt, K. A., et al. 2004, ApJS, 154, 215

Gordon, K. D., Rieke, G. H., Engelbracht, C. W., et al. 2005, PASP, 117, 503

Gordon, K. D., Bailin, J., Engelbracht, C. W., et al. 2006, ApJ, 638, L87

Gordon, K. D., Engelbracht, C. W., Fadda, D., et al. 2007, PASP, 119, 1019

Greenawalt, B., Walterbos, R. A. M., \& Braun, R. 1997, ApJ, 483, 666

Groves, B., Krause, O., Sandstrom, K., et al. 2012, MNRAS, 426, 892

Heesen, V., Krause, M., Beck, R., \& Dettmar, R.-J. 2009, A\&A, 506, 1123

Helou, G., Soifer, B. T., \& Rowan-Robinson, M. 1985, ApJ, 298, L7

Hoernes, P., Berkhuijsen, E. M., \& Xu, C. 1998, A\&A, 334, 57

Hoopes, C. G., \& Walterbos, R. A. M. 2000, ApJ, 541, 597

Hughes, A., Wong, T., Ekers, R., et al. 2006, MNRAS, 370, 363

Krause, M. 2009, in Rev. Mex. Astron. Astrofis. Conf. Ser., 36, 25

Kulsrud, R. M. 2005, Plasma physics for astrophysics (Princeton, NJ: Princeton University Press)

Lacki, B. C., Thompson, T. A., \& Quataert, E. 2010, ApJ, 717, 1

Laine, S., Krause, M., Tabatabaei, F. S., \& Siopis, C. 2010, AJ, 140, 1084

Leroy, A. K., Walter, F., Brinks, E., et al. 2008, AJ, 136, 2782 
Longair, M. S. 1994, High energy astrophysics, 2, Stars, the galaxy and the interstellar medium

Madsen, G. J., Reynolds, R. J., \& Haffner, L. M. 2006, ApJ, 652, 401

Moss, D., Shukurov, A., Sokoloff, D. D., Berkhuijsen, E. M., \& Beck, R. 1998, A\&A, 335, 500

Murphy, E. J. 2009, ApJ, 706, 482

Murphy, E. J., Helou, G., Braun, R., et al. 2006, ApJ, 651, L111

Murphy, E. J., Helou, G., Kenney, J. D. P., Armus, L., \& Braun, R. 2008, ApJ, 678,828

Nesme-Ribes, E., Frick, P., Sokoloff, D., et al. 1995, Academie des Sciences Paris Comptes Rendus Serie B Sciences Physiques, 321, 525

Shalchi, A., ed. 2009, Nonlinear Cosmic Ray Diffusion Theories, Astrophys. Space Sci. Lib., 362

Stanek, K. Z., \& Garnavich, P. M. 1998, ApJ, 503, L131

Stepanov, R., Shukurov, A., Fletcher, A., et al. 2012, MNRAS, submitted [arXiv: 1205.0578]
Strong, A. W., Moskalenko, I. V., \& Ptuskin, V. S. 2007, Ann. Rev. Nucl. Part. Sci., 57, 285

Tabatabaei, F. S., \& Berkhuijsen, E. M. 2010, A\&A, 517, A77

Tabatabaei, F. S., Beck, R., Krause, M., et al. 2007a, A\&A, 466, 509

Tabatabaei, F. S., Beck, R., Krügel, E., et al. 2007b, A\&A, 475, 133

Tabatabaei, F. S., Krause, M., \& Beck, R. 2007c, A\&A, 472, 785

Tabatabaei, F. S., Krause, M., Fletcher, A., \& Beck, R. 2008, A\&A, 490, 1005

Tabatabaei, F. S., Schinnerer, E., Murphy, E. J., et al. 2013, A\&A, 552, A19

Valls-Gabaud, D. 1998, Publ. Astr. Soc. Austr, 15, 111

Viallefond, F., Goss, W. M., van der Hulst, J. M., \& Crane, P. C. 1986, A\&AS, 64, 237

Whitney, B. A., Sewilo, M., Indebetouw, R., et al. 2008, AJ, 136, 18

Xu, C. 1990, ApJ, 365, L47

Yan, H., \& Lazarian, A. 2004, ApJ, 614, 757

Yun, M. S., Reddy, N. A., \& Condon, J. J. 2001, ApJ, 554, 803 